\title{
On the Argument from Physics and General Relativity
}

\section{Christopher Gregory Weaver}

This is a draft of a paper that has been accepted for publication in Erkenntnis. Please cite and quote from the version that will appear in Erkenntnis.

\begin{abstract}
I argue that the best interpretation of the general theory of relativity (GTR) has need of a causal entity (i.e., the gravitational field), and causal structure that is not reducible to light cone structure. I suggest that this causal interpretation of GTR helps defeat a key premise in one of the most popular arguments for causal reductionism, viz., the argument from physics.
\end{abstract}

"The gravitational field manifests itself in the motion of bodies."

- Einstein and Infeld (1949, p. 209)

\section{Introduction}

Causal anti-reductionism or fundamentalism is the view that the causal relation is not grounded in, reduced to, or completely determined by some non-causal phenomenon or phenomena. Causal reductionism is the doctrine that obtaining causal relations are grounded in, reduced to, or completely determined by natural nomicity coupled with the world's unfolding non-causal history. There are two commonly traveled direct paths to causal reductionism. First, one could try to demonstrate that a reductive theory of the causal relation is correct. The accounts of David Fair (1979) and David Lewis (1986a, c) constitute reductive theories. Fair $(1979$, p. 236) argued that causation is nothing over and above the transfer of momentum or energy (a conserved quantity), while Lewis maintained that causation reduces to counterfactual dependence or the ancestral of that relation. ${ }^{1}$ Lewis's complete story characterized counterfactual dependence in terms of the truth of particular counterfactual conditionals whose truth-conditions are strongly related to obtaining degreed similarity relations between possible, albeit, concrete physical worlds. ${ }^{2}$ Laws of nature and the overall history of the involved concrete physical worlds fix the similarity relations. Thus, for Lewis, causation reduces to physical history and natural laws.

Many consider cases of symmetric overdetermination, asymmetric overdetermination, causation by prevention, and causation by omission to be counterexamples to reductive theories. ${ }^{3}$ To illustrate the point by way of just two examples, consider the fact that Fair's view cannot countenance instances of negative causation. ${ }^{4} \mathrm{~A}$ negative causal relation obtains only if, an absence or omission produces an effect. There appear to be actual instances of negative causation involving causation by omission. Problem. How can an absence transfer energy-momentum? The worry is a difficult one. ${ }^{5}$

1 On transference theories generally, see the discussion in Dowe (2000, pp. 41-61). On counterfactual theories generally, see Paul (2009).

${ }^{2}$ See Lewis (1986a,b, c). Lewis would later revise his account (see Lewis (2004)).

${ }^{3}$ For more discussion of these types of cases see Paul and Hall (2013).

${ }^{4}$ See Clarke (2014, pp. 54-58); and Schaffer (2004) on the theme of negative causation.

${ }^{5}$ See the exchange between Dowe (2004) and Schaffer (2004) for a debate about the problem. 
For a second example, suppose, as Lewis (1986a, c) maintained, that $c$ causes $e$, just in case, $e$ and $c$ are distinct events, and $e$ counterfactually depends on $c$ or else there is stepwise counterfactual dependence from $e$ to $c$. The problem of symmetric overdetermination rears its head. Suppose that both $\mathrm{c}_{1}$ and $\mathrm{c}_{2}$ caused $e$, though each was able on their own to causally produce $e$ by themselves. Add that $\mathrm{c}_{l}$ and $\mathrm{c}_{2}$ both occurred at $t$, and both caused $e$ at a time $t+1$. A sufficient actual cause of $e$ was $\mathrm{c}_{l}$, though $e$ does not counterfactually depend upon $\mathrm{c}_{1}$, since had $\mathrm{c}_{1}$ failed to occur, $e$ would have still occurred because $\mathrm{c}_{2}$ would have guaranteed such a fact. Thus, causation and counterfactual dependence come apart and Lewis's $(1986 a, c)$ theory fails. ${ }^{6}$

In spite of such problems, however, reductionists can remain steadfast in their adherence to causal reductionism, for there is a second independent path to that position which travels by way of the argument from physics (unstarred propositions (1)-(3), discussed in sect. 2.1 below). I believe this argument to be unsound, and I support my judgment by arguing that the formalism of GTR ought to be interpreted causally. I suggest that Albert Einstein affirmed a similar interpretation, and (so far as I'm aware) no one has mounted a sustained anti-reductionist defense (that does not require dispositions, or capacities, or causal powers) of what I consider to be the Einsteinian view, despite the fact that the literature is replete with objections to injecting causation into the descriptions and explanations GTR affords. I hope to remedy the situation by defending Einstein (or my interpretation of him), and by responding to many of the philosophical objections.

\subsection{The Roadmap}

Here is my roadmap. First, I formulate the main thesis of the paper in sect. 1. I formulate the argument from physics, and explain how best to defeat one of its key premises in sect. 2. I then employ the strategies outlined in sects. $\mathbf{1}$ and $\mathbf{2}$. to argue, in sect. 3 that what I call minimal fundamentalist causation (explained in sect. 2.3) enters the best interpretation of GTR. In sect. 4, I argue that standard cosmology uses domains of causal influence that help constitute causal pasts and causal futures. I maintain that that causal structure cannot be reduced to or identified with light or null-cone structure. Sect. 5 includes my responses to objections to causal GTR (henceforth C-GTR).

\section{The Argument}

In keeping with remarks previously expressed about the first path to causal reductionism that travels via reductive theories of causation and the independence of the argument from physics, I ask the reader to at least assume that,

Premise 1*: All extant reductive analyses of our causal concept, and all extant reductive theories of the causal relation fail.

182).

${ }^{6}$ This type of counter-example to the counterfactual theory is discussed by Paul (2009, pp. 177- 
Premise $\left(1^{*}\right)$ is generally upheld in our contemporary philosophical milieu, ${ }^{7}$ and I have already sampled why two reductive theories are regarded as problematic in sect. $\mathbf{0}$.

Assuming that extant reductive theories and analyses fail does not by itself privilege causal fundamentalism. All contemporary reductive theories and analyses of causation could fail, and yet causal reductionism come out true. The latter position is not identical to any one reductive analysis or theory. Morevoer, that all contemporary theories fail does not guarantee that there will not be a future successful account.

The strategy behind my affirmation of $\left(1^{*}\right)$ is simple. If your choice reductive theory or analysis were correct, one could always respond to any consideration in favor of admitting causation into any scientific theory whatsoever by reductively analyzing away the involved causal notions or causal structure. I therefore ask the reductionist to check their reductive analyses and theories at the door. The question is whether or not they can justify causal reductionism by way of the argument from physics without using reductive theories or analyses. The question is whether or not the argument from physics can stand on its own.

Premise $2 *$ : If the argument from physics is sound, then all approximately true physical theories require only laws of nature and physical history and do not have need of causation.

This premise follows from the formulation of the argument from physics in sect. 2.1.

Premise $3^{*}$ : The general theory of relativity is an approximately true physical theory whose formalism requires a causal interpretation.

In sect. 2, I show that an interpretation of the formalism of a physical theory like GTR is a part of the theory itself. Sects. 3 and 4 argue that the interpretation of GTR should be causal. I take for granted the fact that GTR is an approximately true physical theory.

Premise $4 *$ : If $(1 *)$ and $(3 *)$, then it is not the case that all approximately true physical theories require only laws of nature and physical history and do not have need of causation.

If my demonstration of $\left(3^{*}\right)$ is successful, and there really is good justification for $\left(1^{*}\right)$, then there is an approximately true physical theory that does not require only laws of nature and non-causal physical history, since it does in fact have need of causation. One is not

${ }^{7}$ The opening page of The Oxford Handbook of Causation (OHC) suggests as much (Beebee, Hitchcock, and Menzies (2009, p. 1)). But see also the remarks in Carroll (2009); Paul and Hall (2013, p. 249); Psillos (2009, p. 154); Schaffer (2007, pp. 872-874); and Tooley (1987, p. 5), inter alios. Carroll (2009, p. 285) notes that the $\mathrm{OHC}$ is filled with papers by reductionists who tell the many stories of failure. Schaffer (2007, pp. 872-874) pleads with his fellow reductionists to abandon the pursuit of reductively analyzing the causal relation. He (2016, sect. 2.1) believes that the argument from physics is what shows that causal reductionism is true. Douglas Kutach (2013, pp. 282-306) tries to show that there probably is no "complete and consistent systematization of cause-effect relations 'out there in reality' that correspond to our folk concept of causation..." (ibid., p. 282) gesturing at the well-known problems and counter-examples to unified theories. 
entitled to interpret the examples of causal structure and causal phenomena in GTR in reductive terms, for that maneuver is precluded by the truth of $\left(1^{*}\right)$. If one needed the truth of a reductive theory of causation so as to escape objections to the argument from physics, it would follow that the soundness of the argument from physics is derivative in that it is dependent upon the success of a reductive theory or analysis of causation. But again, the argument from physics is supposed to be independent motivation for causal reductionism.

Conclusion: Therefore, the argument from physics is unsound.

The conclusion follows logically from the premises.

\section{On Pushing Causation out of Physics and On How to Reintroduce It \\ 2.1. The Argument from Physics}

Channeling, to some degree, Bertrand Russell (1912-1913), Jonathan Schaffer (2008) insisted that there is no room for causation in physical theory. Physics only has need of natural laws and unfolding history. He remarked:

...causation disappears from sophisticated physics. What one finds instead are differential equations (mathematical formulae expressing laws of temporal evolution). These equations make no mention of causation. Of course, scientists may continue to speak in causal terms when popularizing their results, but the results themselves-the serious business of science-are generated independently. ${ }^{8}$

Considerations such as those in the quoted pericope above quite naturally yield the following argument for causal reductionism:

(1) If all approximately true physical theories require only laws of nature and physical history and do not have need of causation, then causal reductionism is true.

(2) All approximately true physical theories require only laws of nature and physical history and do not have need of causation.

(3) Therefore, causal reductionism is true. ${ }^{9}$

This is the argument from physics.

Efforts that consist of justifying premises (1) and (2) are efforts to justify causal reductionism by pushing causation out of physics. I will now turn to the recommendation

${ }^{8}$ Schaffer (2008, p. 92). See also Hall (2011, p. 97); and Hitchcock (2007).

${ }^{9}$ Schaffer (2008, p. 92). The argument Schaffer develops is explicitly one for causal reductionism and not causal eliminativism ( $c f$. ibid., pp. 82-83; p. 86; p. 92). Causal eliminativism is the view that there are no obtaining causal relations (see Russell (1912-1913)).

Schaffer's original argument appealed to scientific practice in general. It is clear, however, that he privileges physics as that science which puts us in touch with the fundamental. Thus, if physics has no need of causation, causal reductionism is well evidenced.

For other arguments in favor of causal reductionism, see Norton (2007a), (2007b). Cf. the recent critical discussion in Frisch (2014, pp. 1-21). 
of a strategy on how to avoid premise (2) by reintroducing causation into successful physical inquiry.

\subsection{How to Defeat the Argument from Physics}

If one assumed that causation is absent from the formalism of a physical theory $\mathrm{T}$, one may still be forced to interpret that formalism causally. As Nancy Cartwright noted in her response to Bas van Fraassen (1989),

The scientific image of nature is no more devoid of cause and causings than is our everyday experience. The appearance to the contrary arises from looking only at science's abstract statements of law, and not how those are used to describe the world. ${ }^{10}$

A predominant way of understanding the structure of $\mathrm{T}$ involves demarcating between its formalism and its interpretation. ${ }^{11}$ The formalism of $\mathrm{T}$ includes mathematical results of the kind with which Schaffer was concerned. In addition, principle-based theories (e.g., some formulations of thermodynamics, and special and general relativity) include in their formalisms underlying principles. Some of these principles are not expressed in a mathematical language at all. A good example of this would be the law of inertia in Newtonian point-mass mechanics of neither the Lagrangian, Hamiltonian, nor axiomatic variety. ${ }^{12}$

An interpretation of $\mathrm{T}$ is a part of $\mathrm{T}$ that specifies how its formalism describes the world (Cartwright (1993, p. 426)). An interpretation of T's formalism will employ T's primitive ideology, or those notions that are indispensable to that interpretation (e.g., the notion of a force in Newtonian mechanics, or energy in classical Lagrangian mechanics, or

${ }^{10}$ Cartwright (1993, p. 426).

11 See Craig (2008, p. 11). Though Frisch (2005, p. 3) does not affirm that the formalism/interpretation distinction is a part of the correct view of theory structure, he calls something like the view I defend the "standard conception".

I will be applying the standard conception to general relativity, but in so doing I do not mean to enter the debate between Curiel (2009) — who argues that GTR does not need an interpretation — on one side, and Belot (1996); and Rovelli (1999)_-who argue that GTR does require an interpretation - on the other. This is because the conception of interpretation in that debate is much broader than what is intended in the present study.

The notion of physical interpretation (the standard conception) I have in mind is closer to Curiel's (2009, p. 46) idea of a categorical interpretation, and it seems almost obvious to me that GTR sits in need of an interpretation in that sense. Indeed, Curiel himself remarked, “...all physical theories have known interpretative problems of the categorical sort..." Curiel (2009, p. 47) emphasis in the original.

My approach to physical interpretations is completely consistent with both the syntactical and semantic views of scientific theory structure. For the syntactic view I have in mind, see Carnap (1967); Hempel (1965, pp. 173-226); and the critical discussion in Suppe (1989, pp. 38-77). Sometimes the syntactic view is called "the received view". On the semantic view, see Giere (1988); Suppe (1989); van Fraassen (1970), van Fraassen (1991, pp. 4-8); van Fraassen (2008, pp. 309-311).

12 The claim that the law of inertia is just $\mathbf{F}=m \mathbf{a}$ for the special case in which $\mathbf{F}=\mathbf{0}$ is demonstrably false. The law of inertia is required to define the entity relative to which the second law holds, viz., an inertial frame ( $c f$. Brown's similar point in his (2005)). 
gravitational field in GTR). ${ }^{13}$ An interpretation will also make explicit use of the ontology of $\mathrm{T}$. The ontology of $\mathrm{T}$ is that list of entities (including structures, processes, properties, and relations) that are required in order to properly connect - by way of a representation relation - the formalism of $\mathrm{T}$ to some proper or improper part of the world (or some suitable possible world). The ontology of $\mathrm{T}$ will also include entities that are indispensable to the direct metaphysical explanation of the truth of the interpreted fundamental laws of T. One popular way to think about metaphysical explanation is in terms of truth-making, where a truthmaker M, makes some proposition true, by de re necessitating its truth. That is to say, M's existence necessitates the truth of the relevant proposition in some non-de dicto fashion. The idea is that T's ontology includes those entities that, in a direct sense (truthmaking may be transitive) make true its fundamental laws.

All of the above breeds a somewhat narrow conception of theories in that different interpretations of the same formalism produce different physical theories. But even after taking on board the narrow conception, one can speak loosely of the general theory of relativity, or non-relativistic quantum theory. A more general use of such locutions may serve historical or pedagogical purposes. In other words, one might, via the locution 'general theory of relativity' intend to pick out that thing which Einstein promulgated in 1915 , or a class of theories that predict and explain virtually all of the same phenomena (e.g., Einstein's GTR and the ADM-formulation of 1962). ${ }^{14}$ These theories* (broad uses of the term will now include asterisks) involve formalisms and partial interpretations of those formalisms. Regarding partial interpretations, Laura Ruetsche wrote,

...the vast majority of the theories philosophers talk about are already partially interpreted. Otherwise they wouldn't be theories of physics. These theories typically come under philosophical scrutiny already having been equipped, by tradition and by lore, with an interpretive core almost universally acknowledged as uncontroversial. ${ }^{15}$

The current project will assume a partial interpretation of GTR* which affirms that the Lorentz metric (i.e., the Lorentzian metric tensor $\mathrm{g}_{a b}$ ) represents the gravitational

${ }^{13}$ Following some related ideas on ideologies of theories in Quine (1951, pp. 14-15); Post (1987); and Hellman and Thompson (1977).

${ }^{14}$ See Arnowitt, Deser, and Misner (2008) for the republication of the original 1962 paper.

${ }^{15}$ Ruetsche $(2011$, p. 7) emphasis in the original. Saunders remarked similarly, “...we may read off the predicates of an interpretation from the mathematics of a theory, and, because theories are born interpreted, we have a rough and ready idea of the objects that they are predicates of." Saunders (2003, pp. 290-291) 
field. ${ }^{16}$ This seems to be the standard view in contemporary relativity physics, ${ }^{17}$ and one could make the case that Einstein's view was close enough to it. ${ }^{18}$ I will also assume that the gravitational field reduces to spacetime geometry. That reduction is also fairly standard in the relativity physics literature. ${ }^{19}$ Thus, if the gravitational field is a causal entity, spacetime geometry should be understood as causally efficacious. As the Class of Physics of the Royal Swedish Academy of Sciences put it, "[t]he new insight [of GTR] was that gravity is really geometric in nature and that the curving of space and time, spacetime, makes bodies move as if they were affected by a force." 20

Completing the partial interpretation of GTR* should involve at least the additional claims that the energy-momentum tensor describes matter fields, and that solutions to Einstein's field equations afford a description of matter, energy, and spacetime geometry. ${ }^{21}$ And so, Einstein and others working in the domain of relativity physics already associated certain interpretive postulates and principles with the relevant formalism. That received "interpretive core" is a part of the extension of more general uses of the locution 'general theory of relativity'. When teaching relativity physics, references to GTR* are often meant to pick out the formalism and accompanying partial interpretation in the early pioneering and contemporary work.

Some may insist that physical theories are in no need of an interpretation because their underlying formalisms are in some way already fully interpreted. But this is not the case. In the context of GTR studies, Carlo Rovelli (1997, pp. 192-195) and Harvey Brown (2005, pp. 159-160; and 2009) argue that the gravitational field is very much akin to a

16 That close association has been challenged in the philosophy of physics literature (see the discussion of the debate in Lehmkuhl (2008); and Rey (2013) and the literature cited therein).

While the view I adopt is similar to Lehmkuhl's (2008, p. 97) "Candidate 3: the metric - and all that it determines", I believe considerations in Lehmkuhl's excellent essay underwrite an inference to the best explanation argument for my assumed partial interpretation. That argument lurks behind the fact that almost all of the mathematical objects in the formalism of GTR thought to describe gravitation derive from the metric tensor. In this sense, their geometric and gravitational significance is parasitic on the geometric and gravitational significance of that sole fundamental tensor in the theory.

${ }^{17}$ See Norton (1989, p. 40) who said that "[i]f we are to call any structure 'gravitational field' in relativity theory, then it should be the metric.” (ibid.); Rindler (2006, p. 179); Rovelli (1997, p. 194); Cf. Torretti (1983, pp. 245-246).

18 Rovelli's (2004, pp. 33-34) remarks assume that the above partial interpretation is a part of Einstein's metrical approach to GTR studies even if that's not the case in quantum gravity studies. Norton (1989, p. 40) says that Einstein "calls the metric tensor the gravitational field." See Einstein (1949, p. 685); Einstein (1952, p. 144); Einstein’s (2002, p. 33).

${ }^{19}$ Carroll (2004, p. 50); Choquet-Bruhat (2009, p. 39); Geroch (2013, p. 65); Hartle (2003, p. 13) says "...the central idea of general relativity is that gravity arises from the curvature of spacetime-the fourdimensional union of space and time. Gravity is geometry." (ibid.) emphasis in the original; Wald (1984, p. 9, cf. p. 68). Weinberg (1972, p. vii) clearly suggests that both (a) the geometrization of gravitation and (b) that the metric tensor represents the gravitational field are standard views (though he seems dissatisfied with at least (a)). Weinberg would go on to say that Einstein adhered to both (a) and (b). See also Rovelli (2004, p. 77). $C f$. Lehmkuhl (2014) for a different perspective.

${ }^{20}$ Class for Physics of the Royal Swedish Academy of Sciences (2011, p. 2).

${ }^{21}$ I'm leaning slightly on Ruetsche (2011, p. 7). I do not whole-heartedly agree with her way of explicating a partial interpretation of GTR* for she injects into the partial interpretation axiomatic formalism (e.g., she explicitly includes Einstein's field equations in GTR's* partial interpretation). For me, the equations are the things being partially interpreted. They are not themselves the substantive content of the (partial) interpretation. 
matter field (like the electromagnetic field), and that gravitational effects are due to that matter-like ${ }^{22}$ field's influence. ${ }^{23}$ That view of the gravitational field is incompatible with the orthodox interpretation that reduces the gravitational field to spacetime geometry itself (q.v., n. 19). For Rovelli and Brown, spacetime does not exist as an independent substance. Thus, substantive interpretive debates enter GTR studies. How one should understand the entities that evolve in accordance with the dynamical formalism is far from obvious.

Return now to the question under consideration: How does one demonstrate that a physical theory like GTR requires causation? Answer: Show that the causal relation is an nonexcludable member of that theory's ontology. Evidence for the thesis that the causal relation is essential to GTR's ontology can issue forth from the fact that the notion of causation shows up indispensably in the best interpretation (its primitive ideology) of the underlying formalism of that theory. Causal GTR (C-GTR) just is a formulation of general relativity whose interpretation entails that GTR's ontology includes (indispensably) the causal relation. But what do I mean by causation? What is the causal relation that I believe is indispensable to GTR like? I will now sketch a general characterization of the type of causation that I believe best suits causal phenomena generally, but general relativistic effects particularly.

\subsection{Which Causation?: The Minimal Fundamentalist Account}

Since we are suppressing, for the purposes of deliberation, the truth of extant reductive analyses and theories of causation, the type of causation at the heart of C-GTR is not a relation that is reducible to counterfactual dependence, probabilistic dependence, the transfer of energy or momentum, or some other reductive surrogate relation or process. Rather, if C-GTR is the best interpretation of GTR's formalism, there will exist in the primitive ideology (the indispensable notions of the ideology) of the theory, a causal notion that refers to a causal relation. That causal relation will be a constituent in the metaphysical explanation of the truth of the interpretation. It will likewise (formally) be necessarily multigrade, asymmetric (though not always temporally asymmetric), transitive, and irreflexive.

Events understood as contingent states of substances exemplifying (joint-carving or scientifically respectable) properties or standing in (joint-carving or scientifically respectable) relations at ontological indices (understood as spacetime locations, or world indices) are the proper relata of causation. Moreover, causation is a dependence relation. When event $x$ causes event $y, y$ depends for its existence and contingent content on $x$. Event $y$ 's contingent content consists of the contingent nexus of exemplification connecting the substance-constituent of $y$ with the properties it exemplifies at the relevant ontological index. If the substance constituent of $y$ is $\sigma$, and we want to know why $\sigma$ has $F$ ness at spacetime location $L$, we can respond with a report on that which caused $y, v i z$., $x$ (given that $x$ satisfies conditions of causal relevance for which see the plausible account of Woodward (2003, pp. 187-238, pp. 351-353)). It is event $x$ 's occurrence that is causally responsible for $y$ 's occurring and for $y$ 's having the contingent content it does $(\sigma$ 's

${ }^{22}$ It is common in general relativity to call electromagnetic radiation a type of matter, though that is not the standard practice in particle physics (Rovelli (1997, p. 219. n. 10)).

${ }^{23}$ Brown (2005, pp. 159-160) seems to be approvingly quoting Rovelli (1997, p. 193), hence my connecting Brown to a view about the gravitational field (as opposed to merely the metric field). 
exemplifying $F$ ness) at the relevant index. Of course, not all causes are causally relevant, but I articulate a case that assumes $x$ is relevant so as to highlight the fact that causation, as I am understanding it, hooks up with causal explanation. Moreover, minimal fundamentalist causation is consistent with multifarious theories of causal explanation (e.g., Strevens (2008); and Woodward (2003)).

Is the above a reductive theory of causation? No. The dependence in play is causal dependence. I therefore agree with Lewis (1986a), (2004), causation should be understood in terms of causal dependence. However, I resist Lewis's additional step of reducing causal dependence to counterfactual dependence. And while this bare bones theory of causation is anti-reductionist/fundamentalist, it is not primitivist. Causal primitivism is the view that causation cannot be accurately described by any theory or analysis that includes entities (including properties and relations), or concepts that are more fundamental than causation itself. The dependence relation at the heart of the phenomenon is itself irreducibly causal. But minimal fundamentalist causation does provide some understanding of the causal relation in terms of something more fundamental, in so far as it seeks to cash out the nature of causation in terms of dependence. What we have here then is an anti-reductionist account of causation that can allow for primitivism about causal dependence though it precludes primitivism about causation. Primitivism about causation entails anti-reductionism about causation, although the converse does not hold (Carroll (2009, p. 281)).

I call the above account, the minimal fundamentalist account. It is fundamentalist because either the involved causal dependence is irreducible and primitive, or else there is some further informative causal characterization of that dependence (i.e., it is not primitive). The latter disjunct is why the account is minimal. It is consistent with more detailed anti-reductionist theories. The fact that $y$ causally depends on $x$ (in the above sense) may itself be grounded in a further fact about manipuability (as in Woodward (2003, pp. 25-93)), or a further fact about how a free agent acting so as to secure the occurrence of $y$ by way of an effective strategy, would use $x$ to bring about $y$ (as in Menzies and Price (1993) these authors regard their position as a reductive one, but that is not the case since they use the notion of a productive agent in their account (cf. Woodward (2003)), or the further fact that some causal law involving a causal necessitation relation between statesof-affair types begins to be instantiated (as in Armstrong (1997, pp. 202-219), given that states of substances as I understand them are Armstrongian concrete token states of affairs). And yet, my theory is incompatible with some powers-based approaches to causation in the work of Mumford and Anjum (2011, 156), and Lowe (2016). While such accounts are anti-reductionist, they reject the tenet that causation is a relation.

Time and space do not permit a full exploration of the minimal fundamentalist account, but suffice it to say that when I affirm that C-GTR is true, that affirmation entails that there are instances of minimal fundamentalist causation in general relativistic effects explained and sometimes described by the dynamics of the theory.

Before passing on to my case for C-GTR, I should highlight that substantiation of the soundness of the main argument that is $\left(1^{*}\right)$-(Conclusion), does not require that I show that minimal fundamentalist causation is instantiated in every single general relativistic effect or dynamical action. Nor does it require that I show that every feature of minimal fundamentalist causation is transparently and expressly manifest in every general relativistic effect or dynamical evolution that I discuss. That burden is overly cumbersome for any proponent of any theory of causation in science. Rather, it is enough to provide an 
abductive case. The best hypothesis that we can proffer about the type of relation or interaction that gravitation is, is the causal hypothesis (i.e., that the gravitational field is a causal entity). As I will show in sect. 3.2, no other relation is fit for the job

\section{The Gravitational Field as Cause}

\subsection{Geodesic Motion in Einstein and Contemporary Physics}

Einstein's general theory of relativity is based upon the strong equivalence principle (SEP) which states that,

In any inertial Lorentz moving frame along a timelike geodesic $\gamma$, all the nongravitational laws of physics, expressed in tensor coordinates with respect to that inertial moving frame, should at each point along $\gamma$ coincide with their special relativistic counterparts expressed in tensor coordinates with respect to global Lorentz frames in Minkowski spacetime. (Prugovečki (1995, p. 52) emphasis removed)

In the standard interpretation, the SEP entails that the metric field structure of spacetime is responsible for inertial and gravitational effects. It implies that gravitation is strongly related to spacetime geometry. ${ }^{24}$ Einstein's field equations (henceforth EFEs, in geometrized units) detail the precise nature of the relationship, ${ }^{25}$

(Eq. 4): $G_{a b} \equiv R_{a b}-\frac{1}{2} R g_{a b}=8 \pi T_{a b}$

Or in SI units and more modern notation with a cosmological constant,

(Eq. $\left.4^{*}\right): R_{\mu \nu}-\frac{1}{2} R g_{\mu \nu}+\Lambda g_{\mu \nu}=\frac{8 \pi G}{c^{4}} T_{\mu \nu}$.

GTR adds to the above formalism (perhaps, merely as theorems), geodesic equations of motion for free and test particles as well as various types of matter. The easiest case is the perfect fluid whose dynamical equation of motion just is,

(Eq. 5): $\nabla^{a} T_{a b}=0$

' $\nabla^{a}$ ' is the covariant derivative operator that satisfies the usual conditions. $T_{a b}=\rho u_{a} u_{b}+$ $P\left(g_{a b}+u_{a} u_{b}\right)$ gives the fluid's energy-momentum tensor. ' $\rho$ ' represents the fluid's density, ' $\mathrm{P}$ ' its pressure, ' $u$ ' its four-velocity, and ' $g_{a b}$ ' is the Lorentz metric representing the gravitational field as in (Eq. 4) (Wald (1984, p. 69)). It is uncontroversial that (Eq. 5) follows from (Eq. 4) (with appropriate interpretive postulates assumed), and there is an

${ }^{24}$ Carroll (2004, p. 50); Janssen (2012, p. 160); Norton (1989, pp. 40-41); Penrose (2005, p. 459). See also Einstein (2002, p. 33). of (Eq. 4).

${ }^{25}$ See Carroll (2004, pp. 155-159) and Wald (1984, pp. 66-74) for clear expositions of the meaning 
associated general principle of geodesic motion (GP) commonly discussed in contexts like these. The GP says that due to gravitational influence, free massive bodies tread down timelike geodesics understood as the straightest possible curves of the spacetime metric. ${ }^{26}$ Or timelike geodesics represent the possible trajectories of free massive bodies that are under the influence of gravitation. The language of influence is no foreign intruder. Physicists typically understand GP that way. Robert J.A. Lambourne wrote,

In general relativity, the time-like geodesics of a spacetime represent the possible world-lines of massive particles falling freely under the influence of gravity alone. And, similarly, the null geodesics of a spacetime represent the possible world-lines of massless particles moving under the influence of gravity alone. ${ }^{27}$

GP has traditionally been interpreted causally, since Einstein and others affirmed that how such objects find themselves in the aforementioned paths is through the determining causal influence of the gravitational field. ${ }^{28}$ Several experts on relativity attest to my reading of Einstein on the matter. Harvey Brown remarked,

...Einstein assumed that all test bodies would follow the grooves or ruts of space-time defined by curves that are straight, or equivalently that are of extremal length. We have seen that during this period Einstein assigned a causal role to space-time structure in precisely this sense: to nudge the particles along such privileged ruts. This kind of action of space-time on matter was taken to be primitive... ${ }^{29}$

Indeed, it would be difficult to explain why Einstein (2013, p. 59) used the actionreaction principle (ARP) to defend GTR, if he did not regard spacetime as a causally

${ }^{26}$ Einstein (2002, p. 339). See the discussion of geodesic equations of motion in Carroll (2004, p. 106-113); Weinberg (1972, pp. 70-73; 121-129); Zee (2013, pp. 275-279; 301-311).

27 Lambourne (2010, p. 137). See also Foster and Nightingale (2005, p. 4); Rindler (2006, p. 178) who says that GTR predicts "the paths of light in [a] vacuum under the influence of gravity."; Romero, Fonseca-Neto, and Pucheu (2011, p. 31).

${ }^{28}$ In 1916, Friedrich Kottler argued that Einstein interpreted the second term of the geodesic equation of motion in such a way that it described the gravitational field's influence on massive bodies. Einstein would recognize this charge in Kottler's work himself (see Einstein (1997, p. 238) I do not understand Einstein's follow-up comment regarding meaninglessness. It does not cohere with what's stated nor with Einstein's general corpus.). He never corrected the causal nature of the interpretation attributed to him (see the interesting discussion of the relevant passages in Lehmkuhl (2014, p. 323)).

Einstein uses quasi-causal language when interpreting the geodesic equation of motion. In Einstein (2002, p. 339), for example, he said the geodesic equation of motion describes "the motion of a material particle under the action only of inertia and gravitation" (ibid. emphasis mine). A little later in the same work Einstein says of the geodesic equation of motion that it expresses "the influence of inertia and gravitation upon the material particle." (ibid., p. 341) emphasis mine.

In dialog with Moritz Schlick about the nature of causation, Einstein (2006) interpreted gravitational phenomena causally.

${ }^{29}$ Brown $(2005$, p. 161). Brown would go on to reject the causal interpretation however. See also Brown and Lehmkuhl (2013, p. 2); Hoefer (2009, pp. 701-702); Pooley (2013, p. 541). Einstein appeared to be both a realist and anti-reductionist about causation (see Ben-Menahem's (1993) discussion). 
efficacious entity. For the ARP says that "a substance is the seat of actions on other substances, and in turn subject to the actions of these other substances..." 30 The application of that principle in this context would not make sense if gravitation were not a causal interaction.

Arguably, one can derive the geodesic equations of motion for massive particles from the EFEs (given certain interpretational postulates). ${ }^{31}$ Causal interpretations of the relevant formalism may therefore be unproblematic for the reductionist, for they can greatly mitigate the evidential importance of those interpretations for the causal reductionism vs. causal anti-reductionism debate by insisting that the theoremhood of those equations/laws suggests that even given a causal interpretation, the involved causal phenomena are not fundamental to GTR or GTR*.

Notwithstanding the easy case of the perfect fluid discussed above, I worry about the relevance and plausibility of many of the derivations for free massive particles in the real world. ${ }^{32}$ Many of them assume an overly idealized or implausible picture of particles. Einstein and Grommer (1927) rejected attempts to derive the equations of motion from the EFEs that appealed to an energy-momentum tensor field $T_{a b}$ description of matter. ${ }^{33}$ To help with attempts at deriving the geodesic equations of motion, they chose to describe matter in terms of singularities. That characterization breeds rather absurd consequences, since on it, geodesics of massive bodies do not reside in space-time (Earman (1995, pp. 1213); Tamir (2012, p. 138 n. 2)). ${ }^{34}$ Other ways of understanding matter in the relevant equations exist, as do other types of attempted proofs that help one skirt around some of the issues. There are, however, potential problems with these alternative approaches (q.v., n. 32). In fact, the problem of articulating exact solutions for two gravitating masses is particularly disturbing. So much so that in 1989 Peter Havas could write,

No...exact solutions can be hoped for in the case of two or more bodies of comparable masses interacting gravitationally...the only exact result to date is a negative one, Weyl's proof of the nonexistence of a static two-body solution. ${ }^{35}$

${ }^{30}$ Brown and and Lehmkuhl (2013, p. 3).

${ }^{31}$ See Eddington (2014, pp. 125-127; $c f$. pp. 149-170); Einstein and Grommer (1927); Einstein, Infeld, and Hoffmann (1938); Einstein and Infeld (1940); Einstein and Infeld (1949); Fock (1959, pp. 215218); Geroch and Jang (1975); Infeld and Schild (1949). The history of attempts to derive the law of motion for particles from the EFEs receives careful attention in Havas (1989); and Havas (1993).

32 For worries about the success and importance of such derivations see Ehlers (1987, pp. 63-65); Tavakol and Zalaletdinov (1998, pp. 312-314, p. 323, p. 325); Tamir (2012).

33 See Einstein (1954, p. 311), and the excerpts from Einstein and Grommer quoted in Havas (1989, pp. 240-241), and Tamir (2012, p. 141).

${ }^{34}$ Einstein admits in a letter to Ludwik Silberstein that "a really complete theory would exist only if the 'matter' could be represented in it by fields and without singularities." As quoted in Havas (1993, p. $102)$ emphasis in the original

${ }^{35}$ Havas (1989, p. 254). Cf. Torretti (1983, p. 177) who says,

"While an exact general solution to the problem of motion in General Relativity is not yet known and may even be impossible, many authors have dealt with it by approximation methods in a variety of cases." (ibid.) 
Suppose there were exact solutions and uncontroversial derivations of the geodesic equations of motion for real particles. What interesting conclusions could we draw? The reductionist could follow Harvey Brown (2005, pp. 161-163). He maintains that plausibility arguments like those in Misner, Thorne, and Wheeler (1973, p. 471-480, henceforth MTW) recommend that the geodesic equations of motion are not axiomatic, and that therefore the geodesic principle should not be understood in causal terms. ${ }^{36}$ I have two responses to Brown's reasoning.

First, if there are successful derivations they would connect field equations with particle behavior. That is initially bizarre. Puzzlement is removed through an appreciation of what the entailment and geodesic equations say. They are not directly and solely about the motions of particles. Rather, one gains insight into the dynamical behavior of the particles (quoting MTW) "by looking at the geometry outside the object[s]. That geometry provides all the handle one needs to follow the motion[s]." ${ }^{37}$ The motions are affected by spacetime geometry, by the behavior of the gravitational field because matter "and the metric are tied together by Einstein's equation". ${ }^{38}$ Gravitational interaction determines geodesic motion. Moreover, departure from geodesics can arise because of features of spacetime geometry. Or, to put it another way, "anomalies in the motion go hand in hand with anomalies in the geometry." 39 These facts only highlight and emphasize the interactive connection between field and particle motion. ${ }^{40}$ In other words, even if one can derive (exactly) the geodesic equations of motion from the EFEs, it is still unclear how precisely the gravitational field couples with matter fields, and it is unclear how the gravitational field influences particles in that the ontology of such coupling and influence is open to interpretation or metaphysical explanation. ${ }^{41}$

Brown's reliance on MTW is, from my standpoint, fortuitous. MTW (1973, pp. 476-477) use causal locutions to interpret the geodesic equations of motion (e.g., "zone of influence" (p. 477)). This supports my contention that, theorem or not, the correct interpretation of the relevant dynamical interaction is causal. The relation that is the interaction between gravitational field and matter fields has a causal ontology. That interpretation is not threatened by recognition of theoremhood. My complete case for this reading is given in sect. 3.2 .

Let me put the two ideas I'm suggesting more explicitly. First, even if the geodesic equations of motion are theorems and not axioms, they both ordinarily are and ought (again see sect. 3.2) to be interpreted causally. If correct, that suggestion is by itself enough to defeat the second premise of the argument from physics. Second, the would-be fact that

36 "The fact that geodesic motion is a theorem and not a postulate has striking consequences that cannot be overemphasized. Earlier...I argued that it (and the need for corrections in the case of bodies with spin) casts doubt on the widespread view that space-time structure, in and of itself, can act directly on test bodies." Brown (2005, p. 162)

${ }^{37}$ Misner, Thorne, and Wheeler (1973, p. 476).

${ }^{38}$ Geroch and Horowitz (1979, p. 212). In the perfect fluid case one clearly sees this in the presence of the Lorentz metric tensor in the energy-momentum tensor of the perfect fluid. Rovelli goes further: “...in all physical equations one now sees the direct influence of the gravitational field...any measurement of length, area or volume is, in reality, a measurement of features of the gravitational field". Rovelli (1999, p. 7)

${ }^{39}$ Misner, Thorne, and Wheeler (1973, p. 476).

${ }^{40}$ See also Nerlich (1976, p. 264).

${ }^{41}$ The coupling talk is particularly relevant for Lagrangian formulations of GTR, which Einstein began to take hold of in 1918. 
the geodesic equations of motion are theorems derived from the EFEs is itself a mathematical fact in need of interpretation. The relevant implication relation suggests an interaction between the dynamical behavior of fields and that of particles. Assenting to the orthodox partial interpretation already outlined above (see sect. 2) allows one to take hold of a dictum that provides a greatly unified and coherent interpretation of GP (at least in part):

(The GP Dictum for Massive Bodies): Necessarily(natural), the gravitational field understood as spacetime curvature causally generates the inertial and time-like geodesic motion of free massive bodies.

It is the truth of (The GP Dictum) that I believe explains the truth of MTW's assertions about spacetime geometry and free massive particle motion.

Second, I disagree with Brown's implicit (2005, p. 161 note the inclusion of “during this period" in his comments on Einstein in ibid.) historical point that Einstein gave up on the causal interpretation once he could derive a geodesic principle from the vacuum EFEs in 1927 (see Einstein and Grommer (1927)). Einstein continued to affirm a causal interpretation of both GP and classical physics more generally. For example, one sees in Einstein's discussion of the relationship between quantum and classical theory in his (1929) essay in Forschungen und Fortschritte a clear commitment to causal structure in the natural world that is described by classical physics, and that will be further revealed by a complete unified field theory. Einstein remarked,

Natural phenomena seem to be determined to such an extent that not only the temporal sequence but also the initial state is fixed to a large extent by law. It seemed to me that I should express this idea by searching for overdetermined systems of differential equations....I strongly believe that we will not end up with a Subkausalität but that, in the indicated sense, we will arrive at an Überkausalität ${ }^{42}$

Pais translates 'Überkausalität', “supercausality”. Einstein's 1929 discussion presupposed a causal understanding of classical physics (see the discussion in Pais (1982, pp. 464-465); Pais (1991, pp. 230-231); cf. the comments on Einstein and causation in quantum physics in Weinert (2005, p. 96)).

Like Einstein, contemporary relativity scholars have not refrained from causally interpreting the action of the gravitational field even after many attempted derivations and plausibility arguments were published. When discussing how precisely to interpret the GP given issues about the argument from physics and causal reductionism in the background,

${ }^{42}$ As quoted and translated by Pais (1982, p. 465). With respect to Einstein and the ultimate unified field theory, Pais (ibid.) goes on to remark, "He [Einstein] demanded that the theory shall be strictly causal, that it shall unify gravitation and electromagnetism, that the particles of physics shall emerge as special solutions of the general field equations..." emphasis mine

Also see his discussion (with Infeld) of the elevator thought experiment in Einstein and Infeld (1938, 226-235). 
Robert Wald remarked that generally, "[t]he metric and matter fields are coupled and undergo causal interactions", and both metric and matter "influence each other causally". ${ }^{43}$

Brown may have been assuming that what is fundamental to a physical theory is that which can be closely read off of the axiomatic formalism of that theory (in this case, the EFEs). But if one goes in for such a view of fundamentality and physical theorizing it ought to be essential to one's understanding of what's fundamental to that theory that everything that is derivative fall out of true descriptions of the world's fundamental structure according to those axiomatic equations and their solutions. God needed only to ensure that a general relativistic spacetime satisfied general relativistic axiomatic equations and/or their solutions, and that certain initial conditions obtained. All other relativistic structure falls out by consequence of the creation of both fundamental structure and initial conditions. This view of the structure of a physical theory does not suit GTR well even though there exists a well-posed initial value formulation of the theory (see Wald (1984, pp. 243-267)). ${ }^{44}$ The geodesic equations of motion for a free particle follow from the EFEs only if certain interpretational postulates are assumed to hold (see on this Malament (2009); and Malament (2012b)). For example, the distributional proofs of the geodesic equation use Einstein's generalized EFEs (Tamir (2012, 144); Infeld (1954); Infeld (1957)). However, the conservation principle $\nabla_{a} T^{a b}=0$ does not follow from those equations, since the Bianchi identities are not true for all solutions to a generalized form of the EFEs since those equations use distributional tensors (Tamir (2012, pp. 143-144) referring explicitly to energy-momentum tensor distributions). However, local energy-momentum conservation is an extremely important principle that does powerful explanatory work in GTR. One must assume it in the context of distributional proofs and even then, there are problems to overcome.

Add that the famous limit operation proof of Geroch and Jang (1975) must use an interpretive postulate in the antecedent of the theorem. As originally stated, the theorem did not use what's called the weak energy condition (i.e., that the energy density of the relevant matter fields is non-negative). ${ }^{45}$ But rather, it made use of (though perhaps inessentially (see the discussion of this in Weatherall (2011)) the strengthened dominant energy condition (though Geroch and Jang called it "the (strong) energy condition" (1975, p. 66)). That condition subsumes the weak energy condition, but adds that the fourmomentum density of the relevant matter fields is a vector that is both timelike and futuredirected ( $c f$. Malament (2012a, 144, or more technically " [g]iven any timelike vector $\xi^{a}$, at any point in $\mathrm{M}, T_{a b} \xi^{a} \xi^{b} \geq 0$ and, if $T_{a b} \neq \mathbf{0}$ there, then $T_{b}^{a} \xi^{b}$ is timelike" (ibid.)).

${ }^{43}$ Robert Wald (personal correspondence, 12/18/2014). See also Geroch (1978, p. 180); Geroch (2013, p. 2, p. 65, p. 68); cf. Misner, Thorne, and Wheeler (1973, pp. 476-477); Nerlich (1994), though not clearly committed to a causal interpretation of spacetime's action says, "[b]ut GR surely makes spacetime something not easily distinguished from a real concrete entity with causal powers" (ibid., p. 183). And Carl Hoefer $(2009$, p. 702) says the causal interpretation is commonly accepted. Brown describes the position that spacetime in GTR acts as a view that is "widespread" (Brown (2005, p. 162)). So far as I'm aware, neither Hoefer nor Brown endorse the causal interpretation I'm defending.

${ }^{44}$ I'm ignoring the problem of specifying "choice" coordinates or a gauge.

${ }^{45}$ More technically, for every timelike vector $\xi^{\mu}$ associated with any point in the differentiable manifold representing spacetime the following relation holds: $T_{\mu \nu} \xi^{\mu} \xi^{\nu} \geq 0$. It is assumed that the energymomentum tensor is appropriately related to the matter fields a hypothetical observer gives attention to. See the discussion in Malament (2009, pp. 6-7); Malament (2012a, p. 144); and Weatherall (2011). 
These energy conditions amount to restrictions on the energy-momentum tensor in the EFEs that do not follow from the EFEs themselves (see the comments of Curiel (2014, p. 41)). They are therefore interpretational postulates not part of the axiomatic equations. They are used to help one understand the nature of the objects described by the axiomatic formalism of GTR.

Perhaps one could incorporate the relevant postulates into the theory as axioms? There are many reasons why that is implausible. Here are two among many. First, it unnecessarily restricts the domain of nomologically possible relativistic spacetimes to those that are described by EFEs with an energy-momentum tensor that satisfies the relevant promoted energy conditions. Second, the energy-momentum tensor depends on, inter alia, the metric $g_{a b}{ }^{46}$ One cannot specify the distribution of matter without determining the metric. The two are intimately related, and one must account for that relationship when seeking to interpret and solve for $T_{\mathrm{ab}}$ (q.v. sect. 5.3 below). ${ }^{47}$ But as I've suggested above and as I will attempt to conclusively argue below, the entity represented by $g_{a b}$ is a causal entity, relating causally to matter distribution. That is the best account. Let me fuel this idea some.

\subsection{The Interaction Must be Causal: Closing the Argumentative Loophole}

In preceding discussion, I argued that Einstein and a great many relativity scholars interpret the geodesic principle causally. I have likewise argued that that interpretation is not defeated by the mere fact that geodesic equations of motion can be derived from the EFEs. The existence of a derivation of the geodesic equations of motion from the EFEs underwrites, and does not count against, the judgment that the gravitational field interacts with matter fields and particles. I will now argue that the causal interpretation is the best interpretation.

\subsubsection{Interactions are in General Causal}

It is a datum that gravitation is an interaction. ${ }^{48}$ Both reductionists and antireductionists affirm that interactions are causal.

In Aronson (1971, p. 421 emphasis mine) a reductive theory is defended, and there exists in that work a choice avenue for specifying causal phenomena in science by way of picking out "changes that result from interactions with other objects" (ibid.). Reductionist Wesley Salmon (1984, with other scholars following him, notably (Hitchcock (2004, pp. 933-934)) constructed his theory of causation by, inter alia, distinguishing between mere happenings such as spatio-temporal intersections, and interactions. The latter were regarded as causal, and the former were judged to be non-causal. When two things were said to interact, (when two footballs collide in mid-air) there is causation. When, however,

${ }^{46}$ Geroch (2013, p. 67); Lehmkuhl (2011, pp. 470-474).

${ }^{47}$ As Geroch noted, "[e]verywhere, we see the metric, directly or indirectly, in the stress-energy...It appears that it is simply impossible to make any reasonable description of matter without the notions of space and time provided by the metric" Geroch (2013, p. 67). See also Hawking and Ellis (1973, p. 61); Malament (2012a, p. 160); Pooley (2013, p. 541. n. 38). The dependence holds even for Lagrangian formulations (see Lehmkuhl (2011, pp. 464-470)). Why is this true? The answer lies in the interpretation of GTR on offer (see sect. 5.3).

48 "Gravity is one of the four fundamental interactions." Hartle (2003, p. 3).

16 ON THE ARGUMENT FROM PHYSICS AND GENERAL RELATIVITY 
two shadows "pass through" each other, there is mere intersection. Whatever one makes of Salmon's heuristic for discerning the difference, or whatever one makes of his view of intersections, it's clear that interactions were univocally regarded as causal. This dogma was not abandoned when Salmon shifted to a conserved quantity theory of causation.

Interactions can be plausibly understood as a species of token instances of interventions as characterized by non-reductive manipulability approaches to causation (for which see Pearl (2009); Spirtes, Glymour, an Scheines (2000); Woodward (2003, pp. 98-151); and Woodward (2009, pp. 243-247) where Woodward explicitly connects total causation and the accompanying account of causal relevance (his condition SC) to a causal interpretation of Newtonian gravitation (ibid., 249)). Many members of this family of views typically understand interventions in terms of "bringing about", or by way of the behavior of "causal mechanisms", or as "actual, and/or direct causation". Pearl explicates causal mechanisms as entities that can interact by way of disruption and interference (see Pearl's remarks in (2009, pp. 225-226); Woodward (2009, pp. 243-244 has a similar reading). Adrian Heathcote (1989) thinks of all causal phenomena as nothing over and above fundamental interactions or interactive phenomena explained and described by quantum field theory. It is clear then that both reductionists and anti-reductionists alike deem interactive phenomena, causal phenomena.

That distinctively physical interactions are causal explains why other interactive theories in the history of physics are standardly outfitted with causal interpretations. For example, in classical Maxwellian electrodynamics, the Lorentz force law $\mathbf{F}=q(\mathbf{E}+$ $\mathbf{v} \times \mathbf{B})$ specifies an interactive relationship between external fields and charged particles. Frisch adds, “...the association between external fields and the acceleration of a charge is usually interpreted causally: External fields cause charges to accelerate" (Frisch (2005, p. 30)). Moreover, the acceleration of a point charge interacts with electromagnetic fields. That is likewise standardly understood in causal terms (Frisch (2005, p. 30)). Our best theory of the strong interaction or strong force is quantum chromodynamics or QCD. That theory is typically interpreted in such a way that the fundamental particles of the theory causally interact with one another by means of undulations of quantum fields (Heathcote $(1989$, p. 100)). Indeed, gluon self-causal interaction is the basis of the theory's asymptotic freedom. Examples and citations could be multiplied.

\subsubsection{Gravitational Interactions are Not Instances of Non-Causal Determination}

Given that gravitation is an interaction, and that interactions are causal, we can mount a strong case for the claim that the causal interpretation of gravitational interaction is indeed forced upon us. We should not, for example, regard the determining influence of the gravitational field as mere non-causal nomological determination, where that relation involves one state of a physical system necessitating another in a way that is backed by dynamical laws (i.e., the dynamical law in question guarantees that the necessitation relation obtains). The "effects abstractly result from certain constellations of temporally prior conditions without necessarily being actively produced by them." 49 There are two reasons for this. First, an appeal to nomological determination begs the question. What

${ }^{49}$ Birnbacher and Hommen (2013, p. 144) emphasis in the original. The authors are there concerned with a reductionist (the laws are non-causal) account of causation whose backbone is essentially the relation of nomological determination. 
ensures that the necessitation relation obtains are the backing laws, and if those laws are causal, they will, with a brand of necessity, guarantee that causes relate to effects. In contrast, the objection on offer has need of non-causal nomological determination relations, and so the laws in play should be non-causal dynamical laws. The backing laws in general relativistic cases will be the laws of GTR. And so, the question just is whether GTR's laws should be interpreted causally. ${ }^{50}$

The most substantial benefit of the causal nomological determination approach is that it fits more appropriately with the datum that gravitation is an interaction. If a physical phenomenon is interactive, then the laws that describe it should be causal. If they are not, then the scientific explanations of general occurrences of that phenomenon that those laws afford will miss out on structure and consequently fail to be complete. My approach is a world-first (or phenomenon-first) natural philosophical approach. We do not learn what the world is like by just looking at disconnected math. There would be no reason for deeming the math approximately correct, if it did not track goings-on in the world. In addition, the math was intended (by Einstein and others) to represent the world. It was intended to explain the perihelion of Mercury, and it was intended to explain why accelerating in a vacuum and "resting" in a uniform gravitational field cannot be discriminated by an observer on operational grounds. Phenomenon first. Math later. That order motivates classes of interpretations of the formalism, in this case, the causal interpretation of GTR. This is because the phenomenon out in the world is universally acknowledged as an interactive one.

There is, of course, a debate in the philosophy of physics literature about whether laws of nature should in general be understood causally. I will not take up the banner of any one side on the more general issue. The specific case of GTR's laws of geodesic motion quite clearly support causal approaches to laws of nature in general relativistic contexts. My judgment is supported by the fact that the leading non-causal approach to laws fails to adequately explain the phenomenon that is the focus of this section. Let's see why by focusing on Robert DiSalle's best-systems treatment of the phenomenon.

\subsubsection{A Mill-Ramsey-Lewis Best-Systems Treatment?}

DiSalle (1995, pp. 323-328) maintained that spacetime structure ought not be regarded as a cause of inertial motion. Rather, there exist non-causal laws of the geometric structure of spacetime which state flatly and brutely that free particles follow geodesics. He said,

When we say that a free particle follows, while a particle experiencing a force deviates from, a geodesic of spacetime, we are not explaining the cause of the difference between the two states or explaining "relative to what" such a difference holds. Instead, we are giving the physical definition of a spacetime geodesic. To say that spacetime has the affine structure thus defined is not to postulate some hidden entity to explain the appearances, but rather to say that

${ }^{50}$ Glennan (2011, p. 811) points out that nomological determination is at home in a causal approach to laws of nature (mentioning the well-known causal account of David Armstrong inter alia). 
empirical facts support a system of physical laws that incorporates such a definition. ${ }^{51}$

DiSalle's take on geodesic motion in relativity is comfortably situated amidst a MillRamsey-Lewis best systems account (BSA) of laws of nature. ${ }^{52}$ This is because what matters for DiSalle is whether the physical theory in question accounts for observations. Puzzlement is removed once one realizes the truth of the definition of geodesic motion and the fact that the system in which that definition is incorporated is sufficiently explanatorily powerful though not at the cost of simplicity. Recall that the BSA affirms that laws of nature constitute a system of propositions that best explains true empirical generalizations in a way that is balanced with simplicity. Ergo, DiSalle's account seems to assume something like the BSA, and so the fate of that account is tied to the plausibility of the BSA or something near enough. This is bad. Independent problems for the BSA abound, ${ }^{53}$ and if that account is for reasons laying outside of GTR false, it should not be invoked here.

Let us set to one side the independent objections to the BSA, and ask what it is specifically about GTR that spells trouble for the BSA. There is an interactive-dynamical explanation of "the appearances" with which DiSalle is concerned. The inertial motion to be explained by the laws, and described by the GP, is not merely "a free particle" following "...a geodesic of spacetime". Rather it is a state involving a gravitating free particle following a geodesic. DiSalle's account fails because it mischaracterizes the geodesic principle, and geodesic motion. It is interesting that so many contemporary statements of the principle remove the language original to it. That language connects geodesic motion to the gravitational field. Einstein's original statement of the principle (what he called the "law of motion") included just such a qualification, for he said that the principle "asserts that a gravitating particle moves in a geodesic line." 54 Thus, DiSalle's best-systems treatment of the laws about the phenomenon of geodesic motion fails to explain what is at issue, the interaction of field and particle.

\subsubsection{Other Treatments?}

Perhaps the relation between gravitational field and matter fields or particles is one of supervenience. The matter fields supervene upon the gravitational field. Of course, more needs to be said, as there are plenty of supervenience relations to go around (e.g., weak, strong, global, mereological, nomological etc.). The most natural choice in this context is nomological supervenience. $J$ property instances nomologically supervene on $K$ property instances, just in case, in the galaxy of naturally possible worlds, $J$ property instances are nomologically necessitated by $K$ property instances. At any world in the relevant galaxy, at the relevant times and locations, a distribution of $K$ property instances (if they exist) ensures a distribution of $J$ property instances at the same times and at or near the same locations in a manner that holds with a kind of natural nomological necessity. Or, to put it

${ }^{51}$ DiSalle (1995, p. 327).

${ }^{52}$ See Lewis (1973, pp. 72-77); Lewis (1983, pp. 365-368); Lewis (1994, pp. 478-482); and Loewer (2012) for clear discussions or presentations of the view.

${ }^{53}$ See particularly the objections in Belot (2011); Hall (2015); and Maudlin (2007).

${ }^{54}$ Einstein (1950, p. 109 emphasis mine). See also Geroch and Jang (1975, p. 65). 
another way, $K$-facts "about a situation naturally necessitate the" $J$-facts. ${ }^{55}$ The idea is that wherever you look out and see in the actual world or (with a modal telescope) in some naturally possible world a certain set arrangement of $K$ property instances you will see the self-same partnering arrangement of $J$ property instances.

Given that nomological supervenience is one's choice relation, the objection from supervenience will beg the question in precisely the same way the invocation of nomological determination did, for as was just explicated above, the standard way to characterize nomological supervenience is in terms of nomological determination or necessitation. ${ }^{56}$

One might forsake supervenience and instead recommend one of the other metaphysically significant relations such as composition, constitution, or realization. Unfortunately, these are, for formal reasons, the wrong relations in this context. Composition, for one thing, is a many-one relation. It is for that, and other obvious reasons disqualified. Constitution is a relation between collocated massive bodies. The gravitational field is not a (traditional) massive body and is not collocated with any massive bodies in cases involving photons (photons are massless). Realization of the kind I favor (see Gillett (2003)) is, like composition, a many-one relation between property-instances "of the parts of a whole, and the properties [or property-instances] of the whole" (modifying Bennett's (2017, p. 11) summary discussion of Gillett), and that disqualifies it in the general relativistic context too.

We are left with grounding. And here I help myself to Jonathan Schaffer's (2009) conception. According to that view, an entity $x$ grounds another entity $y$, just in case, $y$ depends for its positive ontological status (its existence) and nature on $x$ (where the dependence is asymmetric, transitive, and well-founded). I confess that I do not see a reason for distinguishing between causation and grounding in this context. For although Schaffer contrasts grounding priority with causal priority, musing that when $x$ is (in the grounding sense) prior to $y$, it is not causally prior to $y$ (Schaffer (2010, p. 345)), if that suggestion is interpreted as one which claims that causation and grounding can never coincide, the point is made in haste. Surely when (leaning on examples discussed in Schaffer (2000, pp. 165-166)) Merlin casts a spell that transforms a prince into a toad at $t$, he causes a new entity to come into being at $t$. We can say of the toad that it depends for its nature and positive ontological status upon Merlin's spell casting event. It seems then that causal priority can coincide with grounding priority. ${ }^{57} \mathrm{Call}$ such coincident instances of causation and grounding causal grounding.

Return to the case involving the gravitational field and matter fields or particles. Just as in the case of Merlin and spell casting, here too I believe there is causal grounding.

${ }^{55}$ Chalmers (1996, p. 37) although Chalmers calls it natural supervenience.

${ }^{56}$ Again see the characterization in Chalmers (1996, p. 37).

${ }^{57}$ The Merlin case may strike one as too bizarre. Give attention then to any scenario in which something begins to exist on account of a preexisting cause. I, an agent, made a decision to write this paper. Even if agent causation reduces to event causation, and even if mental causation reduces to something noncausal, no problems arise. A plethora of events causally produced a decision, a mental event that was not around before. The decision depends for its existence and nature upon the preceding physical (and perhaps mental) events. If causal reductionism is right, the relevant instance of mental causation reduces to something non-causal, though (importantly) it is not eliminated. Causal reductionism is not causal eliminativism. We have therefore an instance of mental causation, but also an instance of grounding irrespective of whether or not causal reductionism holds. 
If the field's properties cause a particle to have a property (change its trajectory etc.) at some time, then the latter event will depend for its existence and content on the former event as predicted by minimal fundamentalist causation. Schaffer allows for entities from any ontological category to stand in grounding relations.

\section{Domains of Causal Influence in Cosmology: Causal Pasts and Causal Futures}

I have argued that a proper understanding of the gravitational field in GTR implies that causation is indispensable to the best interpretation of GTR's formalism. There is, however, another sense in which causation enters successful physics and that is by way of a proper understanding of the formalism of standard cosmology (which of course subsumes the formalism of GTR, see Weinberg (2008, pp. 1-100; pp. 511-529) for the details). According to standard cosmology, a relativistic spacetime is a pair $\left(M, \mathrm{~g}_{\mathrm{ab}}\right)$, where $M$ is a manifold with four dimensions that is without boundary, and both connected and smooth. The Lorentz metric field $g_{a b}$ is both non-degenerate and smooth. I will also assume that our spacetime is not just time-orientable, but time-oriented.

Given the above assumptions, spacetime points induce double light cone structure. Generally speaking, a light cone is built from a collection of points each of which enjoys a type of connection to the vertex spacetime point of the cone via straight lines that could be traced out by the motions of photons moving away from the vertex spacetime point (leaning on Carroll (2004, p. 9)) if the cone were embedded in spacetime. A double light cone structure involves two such cones that share a vertex point. Our assumptions above allow us to identify one cone in such a structure as the future light cone, and the other the past light cone. Points in the future or past light cone are timelike separated from $p$, points on the cone are light-like separated from $p$, and points outside the cone are spacelike separated from $p$.

There is some confusion about the precise nature of light cone structure in GTR. Some physicists use the locution 'light cone' to refer to a cone structure with vertex point $p$ that lives in a tangent space $\mathrm{V}_{\mathrm{p}}$. Tangent spaces are not spacetime structures (parts of spacetime or representations of spacetime). Tangent spaces are collections of every tangent vector at some spacetime point (Lee (2009, p. 58)). Understood as a space, they are "isomorphic to Minkowski spacetime" (Wald (1984, p. 189)). I will call cone structures in spaces like $\mathrm{V}_{\mathrm{p}}$ null cones. There is another "light cone" structure surrounding spacetime point $p$ that is induced by "null geodesics from p" (Wald (1984, p. 189. n. 1)). I will call this latter type of structure "light cone structure".

Importantly, neither light cones, nor null cones can be identified with causal structure in GTR, where that latter type of structure is what (given our assumptions above) the standard cosmological model associates with spacetime points. That type of structure is sometimes called "domains of influence." Future and past domains of causal influence are represented by the locutions ' $\mathrm{J}^{+}(\mathrm{p})$ ' and ' $\mathrm{J}(\mathrm{p})$ ', where $p$ is a spacetime point that is a

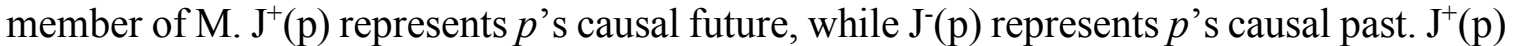
is that "region of space-time which can be causally affected by events in" $p .{ }^{58}$ "Physically,"

${ }^{58}$ Hawking and Ellis (1973, p. 183) emphasis mine. 
(quoting Robert Geroch) $\mathrm{J}^{-}(\mathrm{p})$ "represents the collection of all events of space-time which can affect what happens at $p . " 59$

Quoting scholars who use causal locutions is, of course, not enough for my purposes. But appreciate the further fact that GTR does not reduce or identify domain of influence structure to/with null or light cone structure (e.g., Norton $(2015$, p. 211) denies such identification). First, gravitational lensing precludes the identification of causal structure with light cones. ${ }^{60}$ Second, null cone structure induced by vertex spacetime point $p$ lives in tangent space $V_{p}$, while domains of influence $J+(p)$, and $J-(p)$ are both subsets of the manifold $\mathrm{M}$ itself. They are not in $\mathrm{V}_{\mathrm{p}}$. How can null cones be identical to causal futures and pasts if the two entities live in different spaces? Third, against identification of causal structure and light cone structure, Hawking and Ellis wrote, “...in Minkowski space, the... [light] cone of $p$ forms the boundary of the causal and chronological futures of $p$. However, in more complicated space-times this is not necessarily the case (e.g. see figure 34)." (Hawking and Ellis (1973, p. 184) emphasis mine. Figure 34 references a Minkowski spacetime in which a spacetime point has been removed). ${ }^{61}$

That last point may have been too quick. What I'm arguing is that light cones are not identical to causal pasts/futures. If they were, they'd never come apart. Hawking and Ellis provide a counter-example to the claim that future light cones and causal futures are identical. How do they do this? They provide an example of a spacetime where the two come apart. That spacetime is Minkowskian, and is missing a spacetime point.

What my argumentation shows is that relativity scholars use explicitly causal notions in the ideology of GTR. They give attention to questions about what can causally influence what when using GTR to theorize about the structure of the cosmos. It is therefore

${ }^{59}$ Geroch (2013, p. 123) emphasis mine. Geroch uses ' $\mathrm{I}^{-}(\mathrm{p})$ ' to represent $p$ 's past domain of influence. I am widening Geroch's notion of a domain of influence a bit since even he admits that the term can be misleading, since $\mathrm{I}^{-}(\mathrm{p})$ (having to do with the narrower conception of a past domain of influence) "does not include its boundary, whereas points on the boundary can, in general, also influence p" (ibid., p. 123). I am deliberately intending to include the boundary. In so doing, I follow Bhattacharyya, Colombo, and Sotiriou's (2016, p. 17) identification of domains of influence with causal pasts and futures. Cf. Manchak (2013, p. 590); Ellis and Stoeger (2009).

In this section, I am not arguing that causal structure is causally efficacious. I am not arguing that the specific geometric features standing behind causal structure are themselves causally efficacious. The argument in this section is that there's a type of structure to spacetime that cosmology needs that is causal in that it is explicitly defined in causal terms. That causal taint cannot be dispensed with because that causal structure cannot be identified with any non-causal structure such as light or null cone structure. So the stuff about light/null cones and causal structure is independent of the stuff about the causal efficaciousness of the gravitational field/metric field or spacetime geometry previously discussed in this paper.

${ }^{60}$ Gravitational lensing involving matter or associated caustics can produce a collapse or folding in of the light cone induced by a vertex point $\mathrm{p}$ in the manifold (Ellis, Bassett, and Dunsby (1998, pp. 23462347)); Schutz says “...even a small amount of matter in spacetime will distort light-cones enough to make them fold over on themselves.” (p. 336)). That folding can entail that part of the (past or future) cone bends inside of the causal past or future of p (Perlick (2004); Tavakol and Ellis (1999, p. 41) who include further references on this point). The past/future light cone and the causal past/future of $\mathrm{p}$ cannot therefore be identical. Thanks to George Ellis for help with references.

${ }^{61}$ The mistake of identifying causal structure in relativity with light cone structure is often committed by philosophers (see e.g., Frisch (2014, pp. 16-17); Field (2003, p. 436) comes close to suggesting such identification). Causal or influence structure is standardly regarded as more fundamental than light cone structure in GTR (see Geroch (2013, p. 125)). 
not true that all approximately true physical theories have no need of causation, relativity scholars need causation to carry on with the business of relativity scholarship.

\section{Objections to the Causal Interpretation of GTR}

Causation enters physics by way of the gravitational field and by way of domains of influence. The argument from physics is therefore unsound if the reasoning of sects 3 and 4 is cogent. I will now fend off objections to my case for C-GTR.

\subsection{GTR is Not Fundamental}

Carl Hoefer (2009, pp. 703-704) argues that if GTR implies that there are certain obtaining causal relations, or if its best interpretation makes use of causal notions, the causal reductionist should not be worried, for GTR is not itself a fundamental physical theory. GTR's picture of the world is not the quantum mechanical picture of the world. Perhaps, GTR will have to yield to QM in ways that would rub out any attempt to understand the causal activity of the gravitational field as fundamental physical activity. In response to this, look back to my characterization of Schaffer's argument for causal reductionism. Notice that one of the premises of the argument states that physics can proceed without causation making use instead of natural nomicity and history solely. That premise is not restricted to fundamental physics. GTR is an approximately true and extremely successful physical theory, and that is precisely why any quantum theory of gravity must recover its predictive success. Thus, Hoefer's complaint should not worry the fundamentalist about causation.

It is true that both string theory and loop quantum gravity proponents maintain that GTR should be recoverable within the framework of such quantum theories of gravity in the classical limit. However, there are theories of quantum gravity (QG) that do not seek for such subsumption. The correct theory of QG may be one that is more fundamental than both QM and GTR. Lucian Hardy's causaloid approach to quantum gravity is like this. According to that approach, GTR and QM are "special cases" (Hardy (2007, p. 3082)). Important to Hardy's theory however is indeterminate fundamental dynamical causal structure. ${ }^{62}$ What is more, there are other takes on QG that promote causal structure to fundamental status. For example, causal set approaches to quantum gravity are approaches that, according to Dean Rickles, treat "the causal structure of spacetime as fundamental". ${ }^{3}$ Furthermore, Aron C. Wall (2013) has recently proposed an explicitly causal theory of quantum gravity. The correct framework for a truly quantum theory of gravity is far from settled. The current status of QG studies suggests that any case for the claim "fundamental physics does not need causation" is at best uncertain and incomplete.

${ }^{62}$ Hardy (2007, pp. 3084-3085).

${ }^{63}$ Rickles (2008, p. 347. n. 124) emphasis mine. The quote's immediate context is about Robb's formulation of STR, but it's clear from context that Rickles is also intending to characterize causal set approaches to quantum gravity. 


\subsection{Time-Reversal Invariance and Closed Timelike Curves}

The causal reductionist may still object: The dynamical laws of GTR are timereversal invariant. Therefore, any causal reading of those equations will imply the negation of the principle that necessarily, causes always temporally precede their effects. But surely that principle is true!

The principle that necessarily, causes always temporally precede their effects is in fact false. Recall that in Newtonian mechanics, gravitational interactions obtain instantaneously, and that the gravitational field does not possess a temporal dynamics. ${ }^{64}$ However, the Newtonian gravitational force is commonly understood as causally acting on objects. But if its action does not obey time-governed dynamics, and its interactions are instantaneous, it looks as if simultaneous causation is an implication of Newtonian gravitation. Of course, this response assumes a causal interpretation of Newtonian gravitation, but that interpretation is at least not incoherent. All I need is a possibly true causal interpretation of Newtonian gravitation, and that possible interpretation is Newton's own. Newton said the gravitational force is a centripetal impressed force. Newton took impressed forces to be actions "exerted on a body to change its state either of resting or of moving uniformly straight forward." 65 Newton's more general theory of impressed forces is closely related to the principle that every event or change is caused. ${ }^{66}$

In the Newtonian limit, relativity scholars deal only with small velocities and weak gravitation. It is there that the dynamics of the gravitational field is independent of time (Hawking and Ellis (1973, pp. 71-72); Zee (2013, p. 303) with Zee noting that particles can indeed still move (there's dynamics involved)). It is there that massive bodies can produce gravitational effects in such a way that the dynamics (the gravitation) is described by the Poisson equation $\nabla^{2} \Phi=4 \pi \mathrm{G} \rho$ to which the Einstein field equation reduces (Misner, Thorne, and Wheeler (1973, pp. 415-416)). That equation incorporates a Laplaciean operator with only spatial dimensions (no time). The gravitational (causal) action in the Newtonian limit, even in GTR, appears to be instantaneous though it is not at-a-distance, since the metric field is where the objects are (ibid., 177).

The reductionist may go on to argue that my reading of the dynamical equations implies that backwards causation is possible, since there will be a general relativistic spacetime at which the causes are the effects, and the effects the causes. How can C-GTR employ minimal fundamentalist causation when that causation is asymmetric, and general relativistic action and influence is clearly not?

I believe the objection is confused about the formal property of asymmetry. We say that the causal relation $(C x y)$ is asymmetric, just in case, $\boldsymbol{\nabla} \forall \forall y(C x y \rightarrow \sim C y x)$. In other words, causation is asymmetric if, and only if, necessarily for any $x$ and for any $y, x$ causes $y$, only if, it is not the case that $y$ causes $x$. Given that $x$ actually causes $y$, absolutely no inconsistency with asymmetry arises from the mere fact that at some other possible world, $w, y$ causes $x$. This is because causal asymmetry is not the thesis that necessarily for any $x$ and for any $y, x$ causes $y$, only if, necessarily it is not the case that $y$ causes $x$.

${ }^{64}$ Wald (1984, p. 8); Zee (2013, p. 146).

${ }^{65}$ Newton (1999, p. 405) italics removed.

${ }^{66}$ Jammer (1957, p. 121). Westfall (1971, p. 323). 
The reductionist will continue to push by noting that GTR does not preclude spacetimes with closed timelike curves (CTCs) or closed causal curves (CCCs). If GTR holds, then spacetimes with CTCs are naturally nomologically possible. But it is well known that if spacetimes with CTCs are nomologically possible, then time travel is nomologically possible. If, however, time travel is nomologically possible, then (given the natural necessary falsehood of causal eliminativism) backward causation is naturally possible. Minimal fundamentalist causation is transitive, and asymmetric. That entails that it is also irreflexive. But backwards causation of the kind that involves a body acting at a time $t$, exerting an influence on itself via a causal loop that exploits the exotic causal structure of a spacetime violates irreflexivity (given transitivity). The action and influence in GTR must therefore be non-causal.

I have a two-part response. First, the overlapping and intersecting causal paths provided by CTCs and CCCs are the real cause for worry, not necessarily the phenomenon of backward causation, for there is a defensible position on the matter of CTCs in GTR that preserves the metaphysical possibility of the latter peculiarity while unproblematically abandoning the metaphysical possibility of the former. Here is the idea:

Backwards Causation without Violation of Irreflexivity: The trajectories of particles tracing out part of CTCs or CCCs can be near loops. One need only invoke a spacetime in which a spacetime point is removed, as in spacetimes that violate the strong causality condition (for which see Wald (1984, pp. 196-197)). In such a physically possible world, "there" are "no closed causal curves...but there are causal curves through" spacetime point $p$, say, "which come 'arbitrarily close' to intersecting themselves" (Wald (1984, p. 197)). These spacetimes represent nomological possibilities in which there is genuine backwards causation but no violation of irreflexivity. This is because the involved causal paths do not completely loop back onto themselves thereby creating CCCs or CTCs, though the relevant causal structure does allow for past influence.

The reductionist will ask, is it not unscientific to reject as out of hand or impossible those spacetimes that incorporate CTCs on the basis of a non-scientific principle about causation's formal properties? After all, there are solutions to the EFEs that feature such structure.

Morris, Thorne, and Yurtsever (1988) (MTY) argued that it is nomologically possible that there are wormhole exploiting time machines. Although their study concerned not just general relativity but also quantum field theory, that fact will not rob my illustrative point of all power. So, set it to one side. The history of reactions to MTY is important for acquiring insight into how relativity scholars think about the laws of physics. That history is told well by Kip Thorne (1994, pp. 508-521), who notes how Joseph Polchinski objected to MTY's claim that time travel through wormholes is not paradox-free by envisioning a scenario in which a billiard ball $b$ enters a wormhole $w_{1}$ at some time $t$, exits a suitably connected wormhole $w_{2}$ at an earlier time $t$ - 3 in such a way that it prevents $b$ from ever entering $w_{1}$ by colliding with it. The situation is manifestly paradoxical. If $b$ is prevented from ever entering $w_{1}$, it cannot venture back in time so as to preclude itself from entering the $w_{1}$, though $b$ did enter $w_{1}$. What was inferred from this scenario and from the nature of 
CTCs and time travel in semi-classical contexts (i.e., contexts involving quantized or classical fields, and classical gravitation as in GTR) was that the laws of physics ought to be supplemented with self-consistency principles like the following,

(Self-Consistency Principle (SCP)): If the universe is described by a local solution of the mathematical formalism of the physical laws, then that description is extendable in such a way that it can be a constituent "of a global solution one which is well defined throughout the (nonsingular regions of) classical spacetime." ${ }^{67}$

The SCP is a principle that qualifies the laws of physics. Something like it was introduced (by Friedman et. al. (1990)) to help ensure non-paradoxical evolutions, though those evolutions may very well be consistent with solutions to the formalism of the underlying physical laws. Thus, it seems that SCP's modal strength should at least be as strong as that of nomological necessity so as to help ensure that the laws of the theory that we use to correctly judge some evolution as nomologically possible or impossible do not let in paradoxes. And although John Earman does not think much of time travel paradoxes, he (1995, p. 161, pp. 176-177) argues that consistency constraints ought to be understood as bona fide laws of nature, and that physical possibilities are those possibilities consistent with what are ordinarily considered laws of physics plus consistency principles ${ }^{68}$

I conclude then, that it is not at all unscientific to introduce a nomologically necessary ancillary principle that restricts the space of nomological possibilities to those that are also metaphysically and/or logically possible. Notice that my case for this conclusion remains cogent even if the supposed paradoxes can be resolved. ${ }^{69}$ My conclusion here is that there is nothing unscientific about the strategy, and my justification is that physicists and very scientifically informed philosophers of physics have employed just such a strategy in scientific and philosophical practice.

The question remains. In general relativity, does one encounter paradoxes or impossibilities if one allows for CCCs and CTCs? Since I'm arguing that one should motivate principles that preclude CTCs by appeal to paradoxes implied by them, it will be important to articulate an actual paradox. So here it is.

My articulation of the paradox will assume that at any world at which there exist entities that evolve in accordance with the laws of general relativity, causal eliminativism is false. But I also assume that causal reductionism is true, and that my use of the locution 'cause ${ }_{R}$ ' picks out reductionist causation. Thus, my argument for the paradox begs no questions against causal reductionism. I will also need the posit that causation is transitive.

(1) If GTR without constraining auxiliary principles is true, then it is physically possible that there are closed timelike curves that allow for causal $l_{R}$ loops.

${ }^{67}$ Friedman (et. al.) (1990, p. 1915).

${ }^{68}$ Cf. Stephen Hawking (1992).

${ }^{69}$ Thus, one can endorse the conclusions and resolutions of time travel paradoxes in Earman, Smeenk, and Wüthrich (2009, pp. 93-100) and yet hold on to the supposition that the introduction of principles like $\mathrm{SCP}$ is not unscientific. 
(2) If it is physically possible that there are closed timelike curves that allow for causal $_{\mathrm{R}}$ loops, then an event can cause $\mathrm{R}$ itself.

CTCs that allow for causal $l_{R}$ loops, allow for a causal $R_{R}$ chain that loops back onto itself. If an event A causesR B, and B causes $R$ C, and C causes $R$ A, by transitivity (which we are assuming), A will cause ${ }_{R}$ itself. Thus, if there can be CTCs that allow for causal ${ }_{R}$ loops, then an event (A) can cause $\mathrm{R}_{\mathrm{R}}$ itself.

(3) If an event can cause $e_{R}$ itself, then any event can cause $e_{R}$ itself.

Many reductive theories will come out false if we allow for just a little self-causation . $_{\text {. }}$ However, among those that can tolerate self-causation ${ }_{R}$, perhaps by removing the ' $c$ is not identical to $e$ ' clause in the analyses and/or theories, (3) will come out as a consequence. That is to say, if one's reductive theory does not rule out reflexive causation, then it will probably imply that every event causes $s_{R}$ itself (which entails that any event can cause ${ }_{R}$ itself). For example, consider a regularity account in the tradition of J.L. Mackie's (1965) view, ${ }^{70}$ according to which $c$ causes $e$ if, and only if, $c$ occurs, $e$ occurs, and the proposition that $<c$ occurs. $>$ and the laws together with descriptions of the conditions surrounding $c$ 's occurrence, entails the proposition $<e$ occurs. $>$ But obviously, that will be true in the case in which $c$ is identical to $e$, since it will follow from $<c$ occurs. $>$ alone that $<e$ occurs. $>$, if $c$ is identical to $e$.

(4) It is not the case that any event can cause ${ }_{R}$ itself.

I regard this premise as intuitively obvious.

(5) Therefore, it is not physically possible that there are closed timelike curves that allow for causal $\mathrm{R}_{\mathrm{R}}$ loops, and it is not the case that GTR without constraining auxiliary principles is true. ${ }^{71}$

Thus, the proponent of C-GTR should not worry about CTCs.

\subsection{Back-Reaction and Causal Asymmetry}

John A. Wheeler remarked, "spacetime tells matter how to move; matter tells spacetime how to curve". ${ }^{72}$ If GTR is interpreted causally, then Wheeler's remark will recommend that the gravitational field's behaving $y$-ly causes a material body to behave $x$ ly, while the material body's behaving $x$-ly causes the field to behave $y$-ly. But causation is formally asymmetric. We should therefore enforce a ban on instances of symmetric causation and so on causal GTR.

The gravitational field's dynamical action is primary and causally prior to the inertial motion of massive bodies. There are four reasons that constitute a cumulative case

\footnotetext{
70 This is what Ned Hall (2004, p. 266) calls a "Mackie-style regularity account".

71 The same argument will run with minimal fundamentalist causation in mind.

${ }^{72}$ As quoted by Wheeler (1998, p. 235).
} 
for such a thesis. (a) It is the gravitational field that can enjoy positive ontological status in the absence of matter, and not vice versa. ${ }^{73}$ (b) It is the gravitational field that is fundamental to GTR and not the matter fields (q.v., note 47). (c) It is the gravitational field that can bring about an increase or decrease in the energy-momentum of massive bodies without itself having any localized energy-momentum density, and not conversely. ${ }^{74}$ And (d) it is the gravitational field's direct coupling with matter fields that "results in the latter having an energy tensor", and not contrariwise. ${ }^{75}$ The best explanation of facts (a)-(d) is that the primary causal mover in the context of the inertial motion of massive bodies is the gravitational field and not the matter fields of GTR.

Dennis Lehmkuhl (2011, p. 469) would challenge my appropriation of (d) (and perhaps also the quotation of him for present purposes). ${ }^{76} \mathrm{~A}$ dynamical and causal understanding of (d) is, for Lehmkuhl, incompatible with the fact that tensors of the energymomentum variety are defined in special relativistic contexts too. In STR, the metric $\eta_{\mu \nu}$ isn't a dynamical entity.

On the contrary, there are good reasons for understanding the geometric structure of Minkowski spacetime in STR as dynamical. That is to say, there are good reasons for regarding the geometry of Minkowski spacetime as an indispensable entity in explanations of both the forms of forces acting on objects (their Lorentz invariance), and the behavior of those objects. ${ }^{77}$ Indeed, Einstein went further by insisting that Minkowski spacetime acts. $^{78}$

But a proper response to Lehmkuhl need not go so far. We can instead invoke (noncausal) grounding between facts. Causal facts reporting on the relevant obtaining causal relations are the base, the direct coupling facts (which themselves ground the definitional dependences) are the grounded entities. ${ }^{79} \mathrm{We}$ need not believe that the grounds of an entity

${ }^{73}$ The gravitational field never vanishes even under coordinate transformations, and even in the absence of matter. Moreover, gravitational radiation can propagate in a vacuum.

${ }^{74}$ See Misner, Thorne, and Wheeler (1973, pp. 466-468); Rueger (1998, p. 34).

${ }^{75}$ Lehmkuhl (2011, p. 469). Lehmkuhl is explicitly concerned there with the Lagrangian formulation, for which see Carroll (2004, pp. 159-165).

${ }^{76}$ Lehmkuhl does not deny that there is interaction between metric field and matter in GTR. He said that " ...the matter fields and the metric field $g_{\mu \nu}$ are interacting in GR..." Lehmkuhl $(2011$, p. 469) emphasis in the original.

${ }^{77}$ See the argumentation in Balashov and Janssen (2003, pp. 339-342). Contra Brown (2005); Brown and Pooley (2006); and Craig (2001).

${ }^{78}$ Einstein said,

"That something real has to be conceived as the cause for the preference of an inertial system over a non-inertial system is a fact that physicists have only come to understand in recent years....Also, following the special theory of relativity, the ether [features of Minkowski spacetime geometry] was absolute, because its influence on inertia and light propagation was thought to be independent of physical influences of any kind..." As quoted and translated by Brown and Pooley (2006, p. 68). Einstein said this in 1924.

Brown and Pooley would go on to remark that "[i]t was Einstein's view, then, both that the inertial property of matter can be explained, and that this explanation is to be given in terms of the action of a real entity on the particles". Brown and Pooley (2006, p. 68)

${ }^{79}$ Continuing to use the theory in Schaffer's (2009). 
are essential to it. Thus, the metaphysical explanation of definitional dependence of energymomentum tensor on metric field is, by transitivity, grounded in the fact that the gravitational field causally interacts with the relevant matter fields. At purely STR-worlds, some other ground exists (e.g., conformal features of Minkowski spacetime) for the direct coupling and definitional dependency facts.

Why invoke causation if the direct coupling relations are enough to ground definitional dependency between metric and energy-momentum tensor? The reason lies in what direct coupling amounts to in Lagrangian theories. We say that two (quoting Lehmkuhl) "fields...directly couple if they are factors of the same product term in the Lagrangian, which then gives coupled differential equations as field equations via the Euler-Lagrange equations". ${ }^{80}$ The condition for coupling is couched in terms of a mathematical fact. A Lagrangian is a scalar quantity. Product terms and factors are mathematical entities. What do such mathematical facts suggest about the world? Here I believe is where the causal interaction of the fields has some indispensable grounding role to play. ${ }^{81}$ Interestingly, the metaphysical explanation of the direct coupling facts enters the interpretation of those facts. Thus, if we impugn attributing action to Minkowski spacetime, we can still interpret the Lagrangian formulation of GTR causally, and hold on to fact (d) in the above inference to the best explanation argument.

\subsection{Causal GTR Presupposes Substantivalism}

One might judge that my argumentation essentially requires the truth of spacetime substantivalism. ${ }^{82} \mathrm{I}$ do not think such a position is an essential presupposition of my argumentation, but even if it were, I would not be worried, for substantivalism is the default position in the context of GTR. ${ }^{83}$ Still, spacetime relationalism will not provide an escape from my reasoning. Instead of reducing the gravitational field to spacetime structure thereby attributing dynamical activity to spacetime itself, one could do without an ontological commitment to spacetime understood as an independent substance and treat the metric or gravitational field like a matter field and (perhaps) account for all of the empirical success ordinarily associated with a more orthodox GTR. ${ }^{84}$ I have already mentioned this option in the context of discussing the work of Brown (2005), (2009) and Rovelli (1997)

${ }^{80}$ Lehmkuhl (2011, p. 467) emphasis in the original.

${ }^{81}$ It is important to point out that Lehmkuhl believes that the causal interaction of fields is a sufficient condition for direct coupling (see Lehmkuhl (2011, p. 469)). He too sees room for a relationship between interaction and coupling.

${ }^{82}$ I am assuming that substantivalism is the view that spacetime exists as a substance (quoting Sklar) "independently of the existence of any ordinary material objects, where the latter phrase is taken to include even such extraordinary material objects as rays of light, physical fields...etc." Sklar (1976, p. 161)

On the basis of parsimony motivations, Brown (2005, pp. 24-25) argues against the view that spacetime structure plays an explanatory role in accounting for the motions of bodies. $C f$. the discussion in DiSalle (1994, p. 276); and DiSalle (1995, p. 327).

${ }^{83}$ See the comments in Arntzenius (2012, p. 17 “[a]ccording to...[GTR] spacetime, again, is a single four-dimensional entity." (ibid.)); and Nerlich (2005, p. 281). In general, a realist approach to our most successful theories of physics suggests spacetime substantivalism (see the comments in Pooley (2013, p. 539); and Sklar (1976, p. 214); cf. Weinstein (2001) who argues that relationalism is tough to defend in the context of quantum physics.)

${ }^{84} \mathrm{I}$ do have reservations about such an interpretation. 
at the end of section 1. These two scholars regard the metric or gravitational field as a field akin in nature to matter fields like the electromagnetic field. Belief in the causal action of a matter-like metric or gravitational field does not necessarily commit one to spacetime substantivalism as I have defined it. While Rovelli (2004, p. 78) maintains that the debate between substantivalists and relationalists in the context of GTR is a matter of "semantics" 85 , I believe there remains a substantial dispute. Does there exist an independent substance that is spacetime itself? Does the gravitational field reduce to spacetime geometry? It seems to me that both Brown (2005, p. 156) and Rovelli (2004, p. 77) answer "no" to both questions. For them, there is just the gravitational or metric field, and my point here is that their position is at least consistent with relationalism. Ergo, substantivalism is no essential presupposition of my reasoning.

In point of fact, the matter field approach to gravitation only strengthens the case for the causal interpretation of GTR. The gravitational field can come to possess ripples understood as gravitational waves or gravitational radiation. Gravitational waves propagate, and they are similar to electromagnetic waves in that they exhibit similar causal behavior. Rovelli illustrates the point,

A strong burst of gravitational waves could come from the sky and knock down the rock of Gibraltar, precisely as a strong burst of electromagnetic radiation could. Why is the...[second] 'matter' and the...[first] 'space'? Why should we regard the... [first] burst as ontologically different from the second? Clearly the distinction can now be seen as ill-founded. ${ }^{86}$

We can therefore avoid having to commit to spacetime substantivalism and actually bolster the case for causal GTR if we appropriate one of the best relationalist-friendly approaches to the metric field or gravitational field in general relativistic contexts.

An anonymous referee with Erkenntnis provided me with the following objection. Suppose the Brown-Rovelli matter-like field approach to the gravitational field is wrong, and that the geometric interpretation is right. The following worries ensue,

(W-A): It is false that most affirm both (i) a causal interpretation of the gravitational field's action upon matter and (ii) a geometric interpretation of the gravitational field (i.e., the thesis that the gravitational field reduces to spacetime curvature).

${ }^{85}$ Although later in Rovelli (2004), he seems to think of his choice position as relationalist. He said, “Thus, both GR and QM are characterized by a form of relationalism.” (ibid., p. 220)

${ }^{86}$ Rovelli (1997, p. 193). Weyl compared the gravitational field's action to the productive (causal) behavior of the electric field. He said,

“...just as the electric field, for its part, depends on the charges and is instrumental in producing a mechanical interaction between the charges, so we must assume here that the metrical field...is related to the material content filling the world." Weyl (1952, p. 220) emphasis in the original

In his ontology of physics, Weyl made room for a guidance field that appears to be causal (see Coleman and Korté (2001, p. 198) and the sources cited therein). 
Worry (W-A) is unsurprising because,

(W-B): The geometric interpretation of the gravitational field implies that gravitation is not an interaction, and therefore not causal. ${ }^{87}$

If $\mathrm{W}-\mathrm{B}$ holds, then the argument from interaction in sect. $\mathbf{3}$ will fail, and my primary motivation for C-GTR will be defeated.

In response to $\mathrm{W}-\mathrm{A}$, I remind the reader that I have already provided reasons for believing that both the geometric approach to the gravitational field, and the causal interpretation of GTR are majority views among relativity scholars. It would therefore be surprising if there weren't significant overlap, such that a great many proponents of the geometric approach also affirm a causal interpretation of gravitational action. Second, when one looks to specific relativists who affirm the geometric interpretation, one finds in that same work, again and again, a causal interpretation of GTR and the admission that gravitation is an interaction (see Dodelson (2017, pp. xi-1); Geroch (1978) "[t]he effect of curvature on matter in relativity, via Einstein's equation, is regarded as fundamental by itself." (ibid., 180); for Einstein, again see Pais (1982, p. 465); Einstein and Infeld (1938, pp. 226-235); Hartle (2003, p. 3-4 calling gravitation an interaction on page 3 and then stating on p. 4 that "[g]ravity is geometry." p. 125; Hawking (1996, p. 5); Hawking and Ellis (1973, pp. 1-2, 71); Malament (2012a, p. 121 n. 5, p. 122 n. 6 (approvingly quoting Weyl's causal interpretation), p. 143, p. 146-147, cf. p. 289; Wald (1984, p. 8, p. 71, p. 88; cf. the comments in Pooley (2013, p. 541)). Citations could be easily multiplied.

Worry (W-B) is mistaken. Events are causally efficacious types of entities. I've been working with the view that events are contingent states of substances. It is natural to assume on the geometric approach (though this assumption is not essential to the thesis of the current project) that spacetime is in fact a substance, and that it can have certain properties associated with certain of its regions. These property exemplifications have to do with spacetime's geometry, its curvature, and are bona fide events (spacetime's being a certain way). As such they can and (I have argued) do stand in causal relations. Moreover, it is not as if, on the geometric approach, spacetime curvature fails to influence the rates of clocks, deflect light, constitute ripples that propagate at the speed of light and influence entire galaxies and/or knock down mountains, etc. I'm afraid I do not see any reason why we should abandon the thesis that gravitation ceases to be an interaction on the geometric interpretation of the gravitational field.

${ }^{87}$ The idea that the geometric interpretation precludes a causal understanding of gravitational effects is expressed in many places, but see Livanios $(2008,390)$ who writes, “...[i]t is clear that if we adopt a 4dimensional ontological framework,... and geometrise away the gravitational field, spacetime does not causally affect matter."

I find it interesting that in one of the most exhaustive and most recent discussions of non-causal explanations in science and mathematics (explanations from constraint in Lange (2017)), the section on geometric non-causal explanation (see specifically ibid., 126-128) does not take up these matters having to do with geodesic motion and the gravitational field. Even if it did, Lange's treatise seems most chiefly concerned with explanation. I'm interested in the ontology, what reality needs to be like to back explanations. I happen to think that obtaining causal relations can back non-causal (as well as causal) explanations. In fact, I think certain mathematical truths hold because (this is a because without cause that is indicative of a distinctive non-causal metaphysical explanation such as truth-making or grounding) certain causal relations obtain. Is this not suggested by causally interpreting mathematical formalism? 


\subsection{The Causal Talk is Gloss}

The reductionist may suggest that all of the causal talk I've referenced can be removed without loss of explanatory power. Explicitly causal interpretations of all of the above are therefore problematic. I reply that: Domains of causal influence help determine the global causal structure of spacetime. Without such causal structure one cannot derive the spacetime singularity theorems that are necessary for describing and explaining features of the beginning of the universe (see Wald (1984, p. 188; pp. 237-242) who calls such domains or causal structure a "crucial ingredient" in the proofs of the singularity theorems (ibid., p. 188)).

Perhaps the reason why a reductionist would insist that the causal talk of physicists in general relativistic contexts is gloss is because one can provide a local reduction of causation and causal structure in cosmology and GTR. After all, the problems with many reductive accounts of causation rely on very unique and artificial cases of preemption, overdetermination and the like. Do such cases arise in GTR? The reductionist will bet that they do not. Thus, counterfactual dependence (or some similar reductive surrogate notion) may serve as a worthy proxy for general relativistic causation and causal structure even if it cannot serve as a worthy proxy for causation wherever it is found in the actual world or in broadly logical space.

Local reduction strategies will not work. One can, on paper or with the mind's eye, craft general relativistic worlds at which cases of overdetermination, preemption, and the like occur though these cases involve matter fields and the gravitational field, or gravitational waves, or certain tidal forces. It is particularly easy to get such cases because our world is a general relativistic world. Drop two rocks that are virtually identical at the same time from the exact same height on earth over a rectangular sheet of glass placed just one meter off the ground, and set up the glass in such a way that one of its longest and widest sides is parallel to the ground. If the rocks are dropped high enough, and if the glass is thin enough, each rock will, in a way involving causal overdetermination, bring about the shattering of the glass (hold fixed what you need to in the imagined case). The example involves the gravitational field induced by the earth, and the appropriate matter fields. The example is general relativistic in that it is said to be actual, and the case is one of symmetric overdetermination. This is the same brand of overdetermination that I claimed (in sect. 0) was problematic for counterfactual dependence theories of causation. Thus, general relativistic nomological possibilities and actualities recommend an incompatibility between the local reductive theory of causal structure and GTR itself. A fortiori, the problems with reductive theories of causation are not all revealed in counter-examples or difficult cases. There are many other problems with reductive accounts. ${ }^{88}$

Still causal reductionists will insist that my appeals to GTR and cosmology are cheap and shallow. They will urge that the authorities I have invoked are merely describing matters with a particular gloss. Replace the occurrence of the term 'cause' or 'causal' with some other notion, and all will be well for the argument from physics. But what notion do we replace it with? The phenomenon described is interactive, the relation that obtains between matter fields and gravitational field is one of asymmetric influence (as argued in sect. 5.3) that is present when gravitational waves knock down a mountain (to repeat

${ }^{88}$ See Tooley (2003); and Rueger's (1998) discussion. 
Rovelli's illustrative example from sect. 5.4). The goings-on described are causal by even reductionists lights (as argued in sect. 3.2.1). It seems therefore safe to conclude that in the absence of a truly successful reductive analysis or theory of causation, we have no reason to dispense with causal notions in the primitive ideology of GTR.

\section{Concluding Musings}

I would like to be clear about something I have not done in this paper. I have not argued that causal fundamentalism is true. All of my objections to the argument from physics can be successful and yet causal fundamentalism come out false. One could also be agnostic about the entire debate in question. What I have done is argue that one important and often-traveled path to causal reductionism fails to secure adequate inferential epistemic justification. Such failure is evidence for causal fundamentalism in an important though ultimately inconclusive sense. My arguments are therefore one important first step in a more cumulative case for causal fundamentalism. 
Acknowledgments: I presented earlier versions of this paper at the 2014 Eastern American Philosophical Association conference, Mississippi State University, the University of Illinois at Urbana-Champaign, and Yale University. I thank my audiences at those institutions for their comments. I also thank two anonymous referees with Erkenntnis for their helpful comments and criticisms. The paper has benefited from correspondence with and/or comments from and/or discussions with David Albert, Tom Banks, David Black, Eddy Keming Chen, Shamik Dasgupta, George Ellis, Ned Hall, Michael Townsen Hicks, Barry Loewer, John Norton, Don Page, Laurie Paul, Joshua Rasmussen, Carlo Rovelli, Daniel Rubio, Jonathan Schaffer, Peter van Elswyk, Robert Wald, Aron Wall, and Dean Zimmerman. I thank all of these individuals for their help. Any mistakes that remain are mine. 


\section{References}

Armstrong, D.M. (1997). A World of States of Affairs. New York: Cambridge University Press.

Arnowitt, R. Deser, S. and Misner, C.W. (2008). "Republication of: The Dynamics of General Relativity", General Relativity and Gravitation. 40, 1997-2027.

Arntzenius, F. (2012). Space, Time, and Stuff. New York, NY: Oxford University Press. Aronson, J.L. (1971). "On the Grammar of "Cause"”, Synthese. 22, 414-430.

Balashov, Y. and Janssen, M. (2003). "Review: Presentism and Relativity", The British Journal for the Philosophy of Science. 54, 327-346.

Beebee, H. Hitchcock, C. and Menzies, P. (2009). "Introduction", In Helen Beebee, Christopher Hitchcock, and Peter Menzies (eds.), The Oxford Handbook of Causation. New York: Oxford University Press, 1-18.

Belot, Gordon. (1996). "Why General Relativity Does Need an Interpretation." Philosophy of Science. 63, Supplement: Proceedings of the 1996 Biennial Meetings of the Philosophy of Science Association. Part I: Contributed Papers: S80-S88.

Belot, Gordon. (2011). Geometric Possibility. New York: Oxford University Press.

Ben-Menahem, Y. (1993). "Struggling with Causality: Einstein's Case", Science in Context. 6, 291-310.

Bennett, K. (2017). Making Things Up. New York: Oxford University Press.

Bhattacharyya, J., Colombo, M. and Sotiriou, T.P. (2016). "Causality and Black Holes in Spacetimes with a Preferred Foliation”, Classical and Quantum Gravity. 33, 235003.

Birnbacher, D. and Hommen, D. (2013). "Omissions as Causes - Genuine, Quasi, or Not at All?", In Benedikt Kahmen and Markus Stepanians (eds.), Critical Essays on "Causation and Responsibility". Walter De Gruyter and Co., 133-156.

Brown, H.R. (2005). Physical Relativity: Space-time Structure from a Dynamical Perspective. New York, NY: Oxford University Press.

Brown, H.R. (2009). "The Behaviour of Rods and Clocks in General Relativity, and the Meaning of the Metric Field", arXiv:0911.4440v1 [gr-qc] 23 Nov 2009.

Brown, H.R. and Lehmkuhl, D. (2013). "Einstein, the Reality of Space, and the ActionReaction Principle", arXiv:1306.4902v1 [physics.hist-ph] 20 Jun 2013.

Brown, H.R. and Pooley, O. (2006). "Minkowsi Space-Time: A Glorious Non-Entity", In D. Dieks (ed.), The Ontology of Spacetime. Amsterdam: Elsevier, 67-89.

Carnap, R. (1967). The Logical Structure of the World: Pseudoproblems in Philosophy. Trans. by Rolf A. George. Berkeley, CA: University of California Press.

Carroll, J.W. (2009). “Anti-Reductionism”, In Helen Beebee, Christopher Hitchcock, and Peter Menzies (eds.), The Oxford Handbook of Causation. New York: Oxford University Press, 279-298.

Carroll, S.M. (2004). Spacetime and Geometry: An Introduction to General Relativity. San Francisco, CA: Addison Wesley.

Cartwright, N. (1993). "In Defence of 'This Worldly' Causality: Comments on van Fraassen's Laws and Symmetry", Philosophy and Phenomenological Research. 53, 423-429.

Chalmers, D.J. (1996). The Conscious Mind: In Search of a Fundamental Theory. New York: Oxford University Press.

Choquet-Bruhat, Y. (2009). General Relativity and the Einstein Equations. New York: Oxford University Press. 
Clarke, R. (2014). Omissions: Agency, Metaphysics, and Responsibility. New York: Oxford University Press.

Class for Physics of the Royal Swedish Academy of Sciences. (2011). "Scientific Background on the Nobel Prize in Physics 2011: The Accelerating Universe", URL: http://www.nobelprize.org/nobel_prizes/physics/laureates/2011/advancedphysicsprize2011.pdf

Coleman, R.A. and Korté, H. (2001). "Hermann Weyl: Mathematician, Physicist, Philosopher", In E. Scholz (ed.), Hermann Weyl's Raum-Zeit-Materie and a General Introduction to His Scientific Work. Springer Basel AG, 161-386.

Craig, W.L. (2001). Time and the Metaphysics of Relativity. Philosophical Studies Series 84. Dordrecht: Kluwer Academic Publishers.

Craig, W.L. (2008). "The Metaphysics of Special Relativity: Three Views", In William Lane Craig and Quentin Smith (eds.), Einstein, Relativity and Absolute Simultaneity. New York: Routledge Publishers, 11-49.

Curiel, E. (2009). "General Relativity Needs No Interpretation”, Philosophy of Science. 76, 44-72.

Curiel, E. (2014). “A Primer on Energy Conditions”, arXiv:1405.0403v1 [physics.hist-ph] 30 Apr 2014.

DiSalle, R. (1994). "On Dynamics, Indiscernibility, and Spacetime Ontology”, The British Journal for the Philosophy of Science. 45, 265-287.

DiSalle, R. (1995). "Spacetime Theory as Physical Geometry", Erkenntnis. 42, 317-337.

Dodelson, S. (2017). Gravitational Lensing. New York: Cambridge University Press.

Dowe, P. (2000). Physical Causation (Cambridge Studies in Probability, Induction, and Decision Theory). Cambridge: Cambridge University Press.

Dowe, P. (2004). "Causes are Physically Connected to their Effects: Why Preventers and Omissions are not Causes", In Christopher Hitchcock (ed.), Contemporary Debates in Philosophy of Science. (Contemporary Debates in Philosophy). Malden, MA: Blackwell Publishers, 189-196.

Earman, J. (1995). Bangs, Crunches, Whimpers, and Shrieks: Singularities and Acausalities in Relativistic Spacetimes. New York: Oxford University Press.

Earman, J. Smeenk, C. and Wüthrich, C. (2009). "Do the Laws of Physics Forbid the Operation of Time Machines?”, Synthese. 169, 91-124.

Eddington, A.S. (2014). The Mathematical Theory of Relativity. Originally published Cambridge: Cambridge University Press, 1923. Reprinted Lexington, KY: Bibliolife, 2014.

Ehlers, J. (1987). "Folklore in Relativity and What is Really Known", in M.A.H. MacCallum (ed.), General Relativity and Gravitation: Proceedings of the $11^{\text {th }}$ International Conference on General Relativity and Gravitation. New York, NY: Cambridge University Press, 61-71.

Einstein, A. (1929). "Ansprache von Prof. Einstein an Prof. Planck", Sitzungsberichte Wissenschaftlicher Körperschaften. Forschungen und Fortschritte. Nachrichtenblatt Der Deutschen Wissenschaft und Technik. Fünfter Jahnrgang. 248-249.

Einstein, A. (1949). "Remarks Concerning the Essays Brought Together in this Cooperative Volume", In Paul Aruthur Schilpp (ed.), Albert Einstein: PhilosopherScientist. 665-688. Evanston, IL: The Library of Living Philosophers, Inc. 
Einstein, A. (1950). The Meaning of Relativity. Third Edition, including The Generalized Theory of Gravitation. Trans. by Edwin P. Adams, Appendix I trans. by Ernst G. Straus. Appendix II by Sonja Bargmann. Princeton: Princeton University Press.

Einstein, A. (1952). "The Foundation of the General Theory of Relativity", in H.A. Lorentz, A. Einstein, H. Minkowski, and H. Weyl with notes by A. Sommerfeld and trans. by W. Perrett and G.B. Jeffery, The Principle of Relativity: A Collection of Original Memoirs on the Special and General Theory of Relativity. New York, NY: Dover Publications, Inc., 111-164.

Einstein, A. (1954). Ideas and Opinions. New York, NY: Crown Publishers, Inc. Based on Mein Weltbild edited by Carl Seelig and other sources. New Translations and Revisions by Sonja Bargmann. New York: Bonanza Books.

Einstein, A. (1997). The Collected Papers of Albert Einstein: Volume 6 (English): The Berlin Years: Writings, 1914-1917. (English Translation Supplement) Trans. by Alfred Engel. Engelbert Schucking, Consultant. Princeton, NJ: Princeton University Press.

Einstein, A. (2002). The Collected Papers of Albert Einstein: Volume 7, The Berlin Years: Writings, 1918-1921 English Translation of Selected Texts. English trans. by Alfred Engel. Engelbert Schucking, Consultant. Princeton, NJ: Princeton University Press.

Einstein, A. (2006). "Letter to Moritz Schlick [Berlin,] June 1920", Document 47 In The Collected Papers of Albert Einstein: Volume 10 The Berlin Years: Correspondence, May-December 1920 and Supplementary Correspondence, 1909-1920. Edited by Diana Kormos Buchwald, Tilman Sauer, Ze'ev Rosenkrantz, József Illy, and Virginia Iris Holmes. Trans. by Ann Hentschel. Klaus Hentschel, Consultant. Princeton, NJ: Princeton University Press, 186-187.

Einstein, A. (2013). The Meaning of Relativity: Four Lectures Delivered at Princeton University, May, 1921. Edited by John Gahan, translated by Edwin P. Adams. Princeton: Princeton University Press Reprinted by Desmondous Publications, 2013.

Einstein, A. and Grommer, J. (1927). "Allgemeine Relativitätstheorie und Bewegungsgesetz", Sitzungsberichte der Preussischen Akademie der Wissenschaften, Physikalisch-Mathematische Klasse. 2-13.

Einstein, A. and Infeld, L. (1938). The Evolution of Physics: The Growth of Ideas from Early Concepts to Relativity and Quanta. New York: Simon and Schuster.a

Einstein, A. and Infeld, L. (1940). "The Gravitational Equations and the Problem of Motion II", Annals of Mathematics. Second Series. 41, 455-464.

Einstein, A. and Infeld, L. (1949). "On the Motion of Particles in General Relativity Theory", Canadian Journal of Mathematics. 1, 209-241.

Einstein, A. Infeld, L. and Hoffmann, B. (1938). "The Gravitational Equations and the Problem of Motion", Annals of Mathematics. Second Series, 39, 65-100.

Ellis, G.F.R. Bassett, B.A.C.C. Dunsby, P.K.S. (1998). "Lensing and Caustic Effects on Cosmological Distances", Classical and Quantum Gravity. 15, 2345-2361.

Ellis, G.F.R. and Stoeger, W.R. (2009). "The Evolution of our Local Cosmic Domain: Effective Causal Limits", Monthly Notices of the Royal Astronomical Society. 398, 1527-1536.

Fair, D. (1979). "Causation and the Flow of Energy", Erkenntnis. 14, 219-250. 
Field, H. (2003). "Causation in a Physical World", in Michael J. Loux and Dean W. Zimmerman (eds.), The Oxford Handbook of Metaphysics. New York, NY: Oxford University Press, 435-460.

Fock, V. (1959). The Theory of Space, Time and Gravitation. translated by N. Kemmer. London: Pergamon Press LTD.

Foster, J. and Nightingale, J.D. (2005). A Short Course in General Relativity. Third Edition. New York: Springer.

Friedman, J. Morris, M.S. Novikov, I.D. Encheverria, F. Klinkhammer, G. Thorne, K.S. and Yurtsever, U. (1990). "Cauchy Problem in Spacetimes with Closed Timelike Curves", Physical Review D. 42, 1915-1930.

Frisch, M. (2005). Inconsistency, Asymmetry, and Non-Locality: A Philosophical Investigation of Classical Electrodynamics. New York: Oxford University Press.

Frisch, M. (2014). Causal Reasoning in Physics. Cambridge: Cambridge University Press.

Geroch, R. (1978). General Relativity: From A to B. Chicago, IL: University of Chicago Press.

Geroch, R. (2013). General Relativity: 1972 Lecture Notes. Montreal and Quebec, Canada: Minkowski Institute Press.

Geroch, R. and Horowitz, G.T. (1979). "Global Structure of Spacetimes”, in S.W. Hawking and W. Israel (eds.), General Relativity: An Einstein Centenary Survey. Cambridge: Cambridge University Press, 212-293.

Geroch, R. and Jang, P.S. (1975). "Motion of a Body in General Relativity", Journal of Mathematical Physics. 16, 65-67.

Giere, R.N. (1988). Explaining Science: A Cognitive Approach. Chicago, IL: University of Chicago Press.

Gillett, C. (2003). "The Metaphysics of Realization, Multiple Realizability, and the Special Sciences", The Journal of Philosophy. 100, 591-603.

Glennan, S. (2011). "Singular and General Causal Relations: A Mechanist Perspective", In Phyllis McKay Illari, Federica Russo, and Jon Williamson (eds.), Causality in the Sciences. New York: Oxford University Press, 789-817.

Hall, N. (2004). "The Intrinsic Character of Causation", In Dean W. Zimmerman (ed.), Oxford Studies in Metaphysics. New York: Oxford University Press, 255-300.

Hall, N. (2011). "Causation and the Sciences", in Steven French and Juha Saatsi (eds.), The Continuum Companion to the Philosophy of Science. London: Continuum International Publishing Group, 96-119.

Hall, N. (2015). "Humean Reductionism about Laws of Nature", In Barry Loewer and Jonathan Schaffer (eds.), A Companion to David Lewis (Blackwell Companions to David Lewis). Malden, MA: Wiley Blackwell, 262-277.

Hardy, L. (2007). "Towards Quantum Gravity: A Framework for Probabilistic Theories with Non-fixed Causal Structure", Journal of Physics A: Mathematical and Theoretical 40, 3081-3099.

Hartle, James B. (2003). Gravity: An Introduction to Einstein's General Relativity. San Francisco, CA: Addison Wesley.

Havas, P. (1989). "The Early History of the 'Problem of Motion' in General Relativity", In D. Howard and J. Stachel (eds.), Einstein and the History of General Relativity. Based on the Proceedings of the 1986 Osgood Hill Conference, North Andover, Massachusetts 8-11 May 1986. Boston, MA: Birkhäuser, 234-276. 
Havas, P. (1993). "The General-Relativistic Two-Body Problem and the EinsteinSilberstein Controversy", In J. Earman, M. Janssen, and J.D. Norton (eds.), The Attraction of Gravitation: New Studies in the History of General Relativity (Einstein Studies Volume 5). Boston, MA: Birkhäuser, 88-125.

Hawking, S.W. (1992). "Chronology Protection Conjecture”, Physical Review D. 46, 603611.

Hawking, S.W. (1996). "Classical Theory” In Stephen Hawking and Roger Penrose, The Nature of Space and Time. New Jersey, NJ: Princeton University Press, 3-26.

Hawking, S.W. and Ellis, G.F.R. (1973). The Large Scale Structure of Space-time. (Cambridge Monographs on Mathematical Physics). Cambridge: Cambridge University Press.

Heathcote, A. (1989). "A Theory of Causality: Causality = Interaction (as Defined by a Suitable Quantum Field Theory)", Erkenntnis. 31, 77-108.

Hellman, G. and Thompson, F.W. (1977). "Physicalist Materialism", Nô̂s. 11, 309-345.

Hempel, C.G. (1965). Aspects of Scientific Explanation: And Other Essays in the Philosophy of Science. New York: The Free Press.

Hitchcock, C. (2004). "Causal Processes and Interactions: What Are They and What Are They Good For?”, Philosophy of Science. 71 (5): 932-941.

Hitchcock, C. (2007). 'What Russell Got Right', in H. Price and R. Corry (eds.), Causation, Physics, and the Constitution of Reality: Russell's Republic Revisited. Oxford: Clarendon Press, 45-65.

Hoefer, C. (2009). "Causation in Spacetime Theories", in Helen Beebee, Christopher Hitchcock, and Peter Menzies (eds.), The Oxford Handbook of Causation. New York, NY: Oxford University Press, 687-706.

Infeld, L. (1954). "On the Motion of Bodies in General Relativity Theory", Acta Physica Polinica. 13, 187-204.

Infeld, L. (1957). "Equations of Motion in General Relativity Theory and the Action Principle", Reviews of Modern Physics. 29, 398-411.

Infeld, L. and Schild, A. (1949). "On the Motion of Test Particles in General Relativity", Reviews of Modern Physics. 21, 408-413.

Jammer, Max. (1957). Concepts of Force: A Study in the Foundations of Dynamics. Cambridge, MA: Harvard University Press.

Janssen, M. (2012). "The Twins and the Bucket: How Einstein made Gravity rather than Motion Relative in General Relativity", Studies in History and Philosophy of Modern Physics. 43, 159-175.

Kutach, D. (2013). Causation and its Basis in Fundamental Physics. New York: Oxford University Press.

Lambourne, R.J.A. (2010). Relativity, Gravitation and Cosmology. New York: Cambridge University Press.

Lange, M. (2017). Because without Cause: Non-Causal Explanations in Science and Mathematics. New York: Oxford University Press.

Lee, Jeffrey M. (2009). Manifolds and Differential Geometry. Rhode Island: American Mathematical Society.

Lehmkuhl, D. (2008). “Is Spacetime a Gravitational Field?”, In Dennis Dieks (ed.), The Ontology of Spacetime II (Philosophy and Foundations of Physics) Volume 4. The Netherlands: Elsevier B.V., 83-110. 
Lehmkuhl, D. (2011). "Mass-Energy-Momentum: Only There Because of Spacetime?", The British Journal for the Philosophy of Science. 62, 453-488.

Lehmkuhl, D. (2014). "Why Einstein did not Believe that General Relativity Geometrizes Gravity", Studies in History and Philosophy of Modern Physics. 46, 316-326.

Lewis, D. (1973). Counterfactuals. Malden, MA: Blackwell Publishing.

Lewis, D. (1983). "New Work for a Theory of Universals", Australasian Journal of Philosophy. 61, 343-377.

Lewis, D. (1986a). "Causation”, In David Lewis, Philosophical Papers Volume II, by

David Lewis. New York, NY: Oxford University Press, 159-172.

Lewis, D. (1986b). On the Plurality of Worlds. Malden, MA: Blackwell Publishers.

Lewis, D. (1986c) "Postscripts to 'Causation'." In Philosophical Papers Volume II, by David K. Lewis, 172-213. New York: Oxford University Press.

Lewis, D. (1994). "Humean Supervenience Debugged", Mind 103, 473-490.

Lewis, D. (2004). "Causation as Influence", In John Collins, Ned Hall, and L.A. Paul (eds.), Causation and Counterfactuals. Cambridge, MA: MIT Press, 75-106.

Livanios, V. (2008). "Bird and the Dispositional Essentialist Account of Spatiotemporal Relations", Journal for General Philosophy of Science. 39, 383-394.

Loewer, B. (2012). "Two Accounts of Laws and Time", Philosophical Studies. 160, 115137.

Lowe, E.J. (2016). “There are (Probably) No Relations”, In Anna Marmodoro and David Yates (eds.), The Metaphysics of Relations. Oxford: Oxford University Press, 100112.

Malament, D.B. (2009). "On the Status of the 'Geodesic Law' in General Relativity" Version 2.0, http://philsci-archive.pitt.edu/4908/1/GeodesicLaw.pdf downloaded $12 / 29 / 2015$.

Malament, D.B. (2012a). Topics in the Foundations of General Relativity and Newtonian Gravitation Theory. Chicago, IL: University of Chicago Press.

Malament, D.B. (2012b). "A Remark about the 'Geodesic Principle' in General Relativity", in Mélanie Frapier, Derek H. Brown, Robert DiSalle (eds.), Analysis and Interpretation in the Exact Sciences: Essays in Honour of William Demopoulos. Dordrecht: Springer, 245-252.

Manchak, J.B. (2013). "Global Spacetime Structure", in Robert Batterman (ed.), The Oxford Handbook of Philosophy of Physics. New York, NY: Oxford University Press, 587-606.

Maudlin, T. (2007). The Metaphysics within Physics. New York, NY: Oxford University Press.

Menzies, P. and Price, H. (1993). "Causation as a Secondary Quality", The British Journal for the Philosophy of Science. 44, 187-203.

Misner, C.W. Thorne, K.S. and Wheeler, J.A. (1973). Gravitation. San Francisco, CA: W.H. Freeman and Company.

Morris, M.S. and Thorne, K.S. Yurtsever, U. (1988). "Wormholes, Time Machines, and the Weak Energy Condition", Physical Review Letters. 61, 1446-1449.

Mumford, S. and Anjum, R.L. (2011). Getting Causes from Powers. New York: Oxford University Press.

Nerlich, G. (1976). The Shape of Space. New York: Cambridge University Press.

Nerlich, G. (1994). What Spacetime Explains: Metaphysical Essays on Space and Time. New York: Cambridge University Press. 
Nerlich, G. (2003). "Space-Time Substantivalism", in Michael J. Loux and Dean W. Zimmerman (eds.), The Oxford Handbook of Metaphysics. New York, NY: Oxford University Press, 281-314.

Newton, Isaac. (1999). The Principia: Mathematical Principles of Natural Philosophy. Translated by I. Bernard Cohen and Anne Whitman assisted by Julia Budenz. Preceded by A Guide to Newton's Principia by I. Bernard Cohen. Berkeley and Los Angeles, CA: University of California Press.

Norton, J. (1989). "What was Einstein's Principle of Equivalence?”, In D. Howard and J. Stachel (eds.), Einstein and the History of General Relativity: Einstein Studies Volume 1. Based on the Proceedings of the 1986 Osgood Hill Conference, North Andover, Massachusetts 8-11 May 1986. Boston, MA: Birkhäuser, 5-47.

Norton, J. (2007a). "Causation as Folk Science", in H. Price and R. Corry (eds.), Causation, Physics, and the Constitution of Reality: Russell's Republic Revisited. New York: Oxford University Press, 11-44.

Norton, J. (2007b). "Do the Causal Principles of Modern Physics Contradict Causal AntiFundamentalism?", In P. Machamer and G. Wolters (eds.), Thinking about Causes: From Greek Philosophy to Modern Physics. Pittsburg, PN: University of Pittsburgh Press, 222-234.

Norton, J. (2015). "What Can We Learn about the Ontology of Space and Time from the Theory of Relativity?", in Lawrence Sklar (ed.), Physical Theory: Method and Interpretation. New York, NY: Oxford University Press, 185-228.

Pais, A. (1982). Subtle is the Lord: The Science and the Life of Albert Einstein. New York: Oxford University Press.

Pais, A. (1991). Niels Bohr's Times, In Physics, Philosophy and Polity. Oxford: Clarendon Press.

Paul, L.A. (2009). "Counterfactual Theories”, In Helen Beebee, Christopher Hitchcock, and Peter Menzies (eds.), The Oxford Handbook of Causation. New York: Oxford University Press, 158-184.

Paul, L.A. and Hall, N. (2013). Causation: A User's Guide. Oxford: Oxford University Press.

Pearl, J. (2009). Causality: Models, Reasoning, and Inference. Second Edition. New York: Oxford University Press.

Penrose, R. (2005). The Road to Reality: A Complete Guide to the Laws of the Universe. New York: Vintage Books.

Perlick, V. (2004). "Gravitational Lensing from a Spacetime Perspective", Living Reviews in Relativity. 7 (1): 9.

Pooley, O. (2013). "Substantivalist and Relationalist Approaches to Spacetime", Robert Batterman (ed.), The Oxford Handbook of Philosophy of Physics. New York, NY: Oxford University Press, 522-586.

Post, J.F. (1987). The Faces of Existence: An Essay in Nonreductive Metaphysics. Ithaca, NY: Cornell University Press.

Prugovečki, E. (1995). Principles of Quantum General Relativity. Singapore: World Scientific.

Psillos, S. (2009). "Regularity Theories", In Helen Beebee, Christopher Hitchcock, and Peter Menzies (eds.), The Oxford Handbook of Causation. New York: Oxford University Press, 131-157. 
Quine, W.V.O. (1951). “Ontology and Ideology”, Philosophical Studies. 2, 11-15.

Rey, D. (2013). "Similarity Assessments, Spacetime, and the Gravitational Field: What

Does the Metric Tensor Represent in General Relativity?", http://philsciarchive.pitt.edu/id/eprint/9615 Foundations of Physics 2013: The $17^{\text {th }} \mathrm{UK}$ and European Meeting on the Foundations of Physics (Munich; 29-31 July 2013).

Rickles, D. (2008). "Quantum Gravity: A Primer for Philosophers”, in Dean Rickles (ed.),

The Ashgate Companion to Contemporary Philosophy of Physics. Aldershot and Burlington: Ashgate Publishing Limited and Ashgate Publishing Company, 262-383.

Rindler, W. (2006). Relativity: Special, General and Cosmological. $2^{\text {nd }}$ Edition. New York: Oxford University Press.

Romero, C. and Fonseca-Neto, J.B. and Pucheu, M.L. (2011). "General Relativity and Weyl Frames", International Journal of Modern Physics: Conference Series. 3, 2735.

Rovelli, C. (1997). "Halfway through the Woods: Contemporary Research on Space and Time”, In John Earman and John D. Norton (eds.), The Cosmos of Science: Essays of Exploration. Pittsburg, PN: University of Pittsburg Press, 180-223.

Rovelli, C. (1999). “Quantum Spacetime: What Do We Know?”, arXiv:gr-qc/9903045v1 12 Mar 1999.

Rovelli, C. (2004). Quantum Gravity (Cambridge Monographs on Mathematical Physics). New York, NY: Cambridge University Press.

Rueger, A. (1998). "Local Theories of Causation and the a posteriori Identification of the Causal Relation", Erkenntnis. 48, 25-38.

Ruetsche, L. (2011). Interpreting Quantum Theories: The Art of the Possible. New York, NY: Oxford University Press.

Russell, B. (1912-1913). 'On the Notion of Cause', Proceedings of the Aristotelian Society. 13, 1-26.

Salmon, W.C. (1984). Scientific Explanation and the Causal Structure of the World. Princeton, NJ: Princeton University Press.

Saunders, S. (2003). "Physics and Leibniz's Principles." In Katherine Brading and Elena Castellani (eds.), Symmetries in Physics: Philosophical Reflections. Cambridge; Cambridge University Press, 289-307.

Schaffer, J. (2000). "Trumping Preemption", The Journal of Philosophy. 97, 165-181.

Schaffer, J. (2004). "Causes Need not be Physically Connected to their Effects: The Case for Negative Causation", In Christopher Hitchcock (ed.), Contemporary Debates in Philosophy of Science (Contemporary Debates in Philosophy). Malden, MA: Blackwell Publishing, 197-216.

Schaffer, J. (2007). "Review: Dowe and Noordhof, Cause and Chance", The British Journal for the Philosophy of Science, 58, 869-874.

Schaffer, J. (2008). 'Causation and Laws of Nature: Reductionism', in Theodore Sider, John Hawthorne, and Dean W. Zimmerman (eds.), Contemporary Debates in Metaphysics (Contemporary Debates in Philosophy) Malden, MA: Blackwell Publishers, 82-107.

Schaffer, J. (2009). "On What Grounds What", In David J. Chalmers, David Manley, and Ryan Wasserman (eds.), Metametaphysics: New Essays on the Foundations of Ontology. New York: Oxford University Press, 347-383.

Schaffer, J. (2010). "The Internal Relatedness of All Things", Mind. 119, 341-376. 
Schaffer, J. (2016). "The Metaphysics of Causation", In Edward N. Zalta (ed.), The Stanford Encyclopedia of Philosophy (Fall 2016 Edition). https://plato.stanford.edu/archives/fall2016/entries/causation-metaphysics/

Schutz, B. (2003). Gravity from the Ground Up: An Introductory Guide to Gravity and General Relativity. New York: Cambridge University Press.

Sklar, L. (1976). Space, Time, and Spacetime. Berkely and Los Angeles, CA: University of California Press.

Spirtes, P. Glymour, C., and Scheines, R. (2000). Causation, Prediction and Search. $2^{\text {nd }}$ Edition. Cambridge, MA: MIT Press.

Suppe, F. (1989). The Semantic Conception of Theories and Scientific Realism. Urbana and Chicago, IL: University of Illinois Press.

Tamir, M. (2012). "Proving the Principle: Taking Geodesic Dynamics Too Seriously in Einstein's Theory", Studies in History and Philosophy of Modern Physics. 43, 137154.

Tavakol, R. and Ellis, G. (1999). "Holography and Comsology", Physics Letters B. 469, 37-45.

Tavakol, R. and Zalaletdinov, R. (1998). "On the Domain of Applicability of General Relativity", Foundations of Physics. 28, 307-331.

Thorne, K.S. (1994). Black Holes and Time Warps: Einstein's Outrageous Legacy. New York: W.W. Norton \& Company.

Tooley, M. (1987). Causation: A Realist Approach. New York, NY: Oxford University Press.

Tooley, M. (2003). "Causation and Supervenience", in Michael J. Loux and Dean W. Zimmerman (eds.), The Oxford Handbook of Metaphysics. New York, NY: Oxford University Press, 386-434.

Torretti, R. (1983). Relativity and Geometry. New York: Pergamon Press.

van Fraassen, B.C. (1970). "On the Extension of Beth's Semantics of Physical Theories," Philosophy of Science. 37, 325-339.

van Fraassen, B.C. (1989). Laws and Symmetry. New York, NY: Oxford University Press.

van Fraassen, B.C. (1991). Quantum Mechanics: An Empiricist View. Oxford: Clarendon Press.

van Fraassen, B.C. (2008). Scientific Representation: Paradoxes of Perspective. New York: Oxford University Press.

Wald, R.M. (1984). General Relativity. Chicago, IL: University of Chicago Press.

Wall, A.C. (2013). “A Discrete, Unitary, Causal Theory of Quantum Gravity”, Classical and Quantum Gravity. 30, 115002.

Weatherall, J.O. (2011). "A Brief Remark on Energy Conditions and the Geroch-Jang Theorem”, arXiv:1106.2336v1 [physics.hist-ph] 12 Jun 2011.

Weinberg, S. (1972). Gravitation and Cosmology: Principles and Applications of the General Theory of Relativity. New York: John Wiley \& Sons, Inc.

Weinberg, S. (2008). Cosmology. New York, NY: Oxford University Press.

Weinert, F. (2005). The Scientist as Philosopher: Philosophical Consequences of Great Scientific Discoveries. Berlin: Springer-Verlag.

Weinstein, S. (2001). “Absolute Quantum Mechanics”, The British Journal for Philosophy of Science 52, 67-73. 
Westfall, R.S. (1971). Force in Newton's Physics: The Science of Dynamics in the Seventeenth Century. New York, NY: American Elsevier.

Weyl, H. (1952). Space-Time-Matter. Fourth Edition. Translated by Henry L. Brose. Mineola, NY: Dover Publications.

Wheeler, J.A. (1998). With Kenneth Ford. Geons, Black holes, and Quantum Foam: A Life in Physics. New York, NY: W.W. Norton and Company, Inc.

Woodward, J. (2003). Making Things Happen: A Theory of Causal Explanation. New York: Oxford University Press.

Woodward, J. (2009). “Agency and Interventionist Theories.” In The Oxford Handbook of Causation, edited by Helen Beebee, Christopher Hitchcock, and Peter Menzies, 234262. New York: Oxford University Press.

Zee, A. (2013). Einstein Gravity in a Nutshell. Princeton, NJ: Princeton University Press. 